MATHEMATICS OF COMPUTATION

Volume 76, Number 259, July 2007, Pages 1163-1194

S 0025-5718(07)01948-5

Article electronically published on February 7, 2007

\title{
NONLINEAR PROJECTION METHODS FOR MULTI-ENTROPIES NAVIER-STOKES SYSTEMS
}

\author{
CHRISTOPHE BERTHON AND FRÉDÉRIC COQUEL
}

\begin{abstract}
This paper is devoted to the numerical approximation of the compressible Navier-Stokes equations with several independent entropies. Various models for complex compressible materials typically enter the proposed framework. The striking novelty over the usual Navier-Stokes equations stems from the generic impossibility of recasting equivalently the present system in full conservation form. Classical finite volume methods are shown to grossly fail in the capture of viscous shock solutions that are of primary interest in the present work. To enforce for validity a set of generalized jump conditions that we introduce, we propose a systematic and effective correction procedure, the so-called nonlinear projection method, and prove that it preserves all the stability properties satisfied by suitable Godunov-type methods. Numerical experiments assess the relevance of the method when exhibiting approximate solutions in close agreement with exact solutions.
\end{abstract}

\section{INTRODUCTION}

The present work treats the numerical approximation of the solutions of the Navier-Stokes equations for a compressible fluid modelled by $N, N \geq 2$, independent pressures. By independent, we mean that the pressures come with their own specific entropy and must be thus governed by $N$ independent entropy balance equations. Then the sum of all the pressures yields the total pressure in the fluid.

Despite that such PDE models are seen hereafter to exhibit several close relationships with the usual Navier-Stokes equations, the fundamental discrepancy stays in the lack of an admissible change of variables that recasts the present $(N+2) \times(N+2)$ system in full conservation form. Indeed, the three classical conservation laws ruling density, momentum and total energy must be supplemented by $N-1$ of the entropy balance equations that govern the $N$ pressures. However, none of these entropy balance equations boils down to a conservation law without restrictive modelling assumptions to be put on the $N$ viscosity laws. These equations generally involve nonconservative products that account for dissipative phenomena: namely the entropy dissipation rates.

Such systems can be understood as a natural extension of the classical NavierStokes equations, i.e., equipped with a single entropy balance equation, in that

Received by the editor March 2, 2005 and, in revised form, April 16, 2006.

2000 Mathematics Subject Classification. Primary 65M99, 65M12; Secondary 76N15.

Key words and phrases. Navier-Stokes equations, entropy inequalities, nonconservative products, travelling wave solutions, Godunov-type methods, discrete entropy inequalities, nonlinear projection, turbulence models. 
they actually occur in several distinct settings from physics. They arise for instance in plasma physics where the electron temperature must be distinguished from the mixture temperature of the other (heavy) species (see [6] for instance). After Berthon [1, they are also recognized under that form within the framework of the so-called "two transport" equations models for turbulent compressible flows where the averaged thermodynamic temperature must be distinguished from the specific turbulent kinetic energy $k$. The comprehensive study 1 actually shows that the $(k, \epsilon),(k, \omega)$ or $(k, l)$ models of prominent practical importance enter the present framework (see [18, 19, 20], 24] for a derivation from physics). An important issue is that all these models are usually addressed with the assumption of a very large Reynolds number. From a practical point of view, the Reynolds number is typically larger than $10^{6}$.

The numerical capture of the viscous shock layers coming with the Navier-Stokes extended system is the heart of the present work. Since the Reynolds numbers of interest are large, these layers display the character of a shock wave in that they differ from their end states only in a small interval of rapid transition. Hence for mesh refinements of practical interest, the associated discrete profiles stay largely underresolved (see Figure 1 for the negative consequences within the framework of a classical splitting numerical method that we briefly describe hereafter). Our purpose is actually to properly capture the two end states, $\mathbf{u}_{L}$ and $\mathbf{u}_{R}$, of a given shock layer together with its relevant speed of propagation $\sigma$ without resolving the viscous layer itself. The correct prediction of these end states should be achieved independently of the mesh refinement as far as possible.

It is quite well known that such an issue does not raise special difficulties within the standard framework of the Navier-Stokes equations, e.g., in conservation form. Indeed, the triples $\left(\sigma ; \mathbf{u}_{L}, \mathbf{u}_{R}\right)$ are solutions of the classical Rankine-Hugoniot relations (see Gilbarg [10]), and the celebrated finite volume methods in conservation form readily ensure their suitable capture (see Lax-Wendroff [16]). At the very core of this success is that the end states just solve the usual jump conditions that are obviously free from the underlying viscous regularization: i.e., the precise definition of (say) the right end state $\mathbf{u}_{R}=\mathbf{u}_{R}\left(\sigma ; \mathbf{u}_{L}\right)$ stays free from the exact modelling of the diffusive phenomena. For this reason and loosely speaking, the numerical capture of this end state stays fairly free from the exact form of the numerical dissipation artificially induced by a scheme in conservation form.

The situation is however completely different in the setting of the extended Navier-Stokes system that we tackle. Its nonconservation form makes this time the exact definition of the end state $\mathbf{u}_{R}\left(\sigma ; \mathbf{u}_{L}\right)$ to heavily depend on the precise shape of the diffusive tensor, since there no longer exists a full set of classical jump conditions. The missing information in the jump is primarily dictated by the underlying viscous regularization. The reader can consult at this stage Figure 2 for convincing numerical illustrations reporting the strong sensitiveness of exact end states $\mathbf{u}_{R}\left(\sigma ; \mathbf{u}_{L}\right)$ to the ratio of viscosities entering the present extended Navier-Stokes model. Let us underline that such a dependence stays at the basis of recent works devoted to hyperbolic systems involving nonconservative products (see LeFloch [17, Dal Maso-LeFloch-Murat [8, Raviart-Sainsaulieu [21]). When rephrased in the setting of numerical methods, this sensitivity translates into a heavy dependence of the approximate solutions to the underlying artificial viscosity. Loosely speaking, 

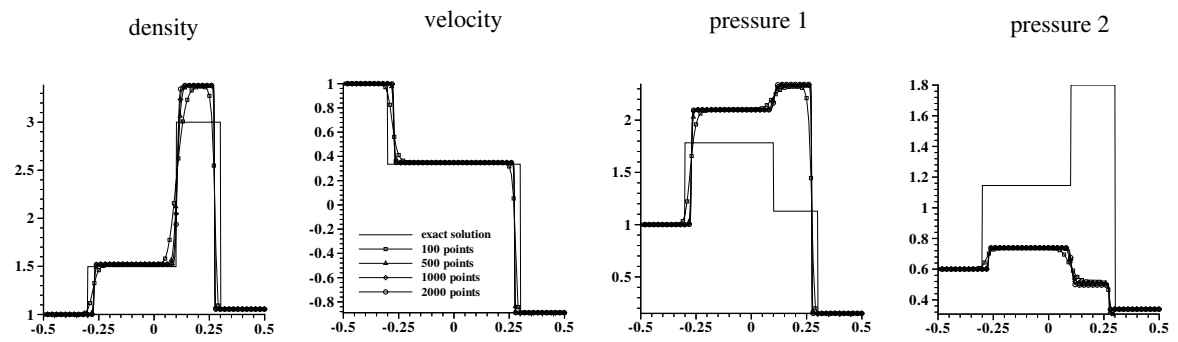

Figure 1. Typical failure of classical finite volume methods in the capture of viscous shock layers

expecting a good agreement with exact solutions urges the numerical viscosity matrix entering the discrete method (see Tadmor [26]) to fit in some sense the exact diffusive tensor involved in the PDEs. Such a vague sense has received a theoretical foundation in a work by Hou and LeFloch [12] (see also [4 for a complementary standpoint) and is actually the starting point of some numerical methods as devised by Karni [13, for instance. In this work, fitness is measured via a modified equations approach according to which unsuited truncation errors are forced to cancel up to a given order. Motivated by Hou and LeFloch [12, we propose a powerful and complementary approach based on the analysis of the discrete entropy dissipation rates coming with a given numerical method. We refer the reader to Tadmor [26] for a precise link between the numerical viscosity matrix and the discrete entropy rate. We exhibit in the present setting underlying generalized jump conditions, in sufficient number, expressing a set of $N-1$ key balance properties between each of the $N$ entropy dissipation rates. When added to the three usual jump relations for density, momentum and total energy, they yield a complete set of conditions but relating $N+3$ quantities in their jump for only $N+2$ independent variables. The additional quantity is nothing but a Lax mathematical entropy for the extended Navier-Stokes system. As we prove hereafter, the refinement-free capture of the end states of viscous shock layers requires the exact preservation of this complete set of generalized jump conditions. At the discrete level, we have to face the nonstandard and difficult issue of controlling a prescribed dissipation rate in the additional Lax entropy. In order to illustrate the negative consequences of a failure in the above requirement, let us consider the numerical results displayed in Figure 1. These are obtained using a classical splitting method briefly derived in the forthcoming numerical section. This problem enters the present setting for extended Navier-Stokes equations with $N=2$ when choosing two strictly positive viscosity coefficients. The discrete solutions seem to coincide with a given function. However such a function exhibits unacceptable errors with the exact solution plotted in solid lines. Let us underline that the two extreme discontinuities in the approximate solutions formally coincide with two viscous shock layers. In agreement with our main purpose, these layers are underresolved for the mesh refinements under consideration.

For the sake of simplicity in the notation, we focus ourselves in the sequel to the case of two independent specific entropies. The nonlinear projection method we propose will be seen to easily extend to additional independent entropies. 
The format of the present paper is as follows. The second section describes the extended Navier-Stokes system, paying attention to the main properties of its travelling wave solutions (i.e. viscous shock layers). A close characterization of the triples $\left(\sigma ; \mathbf{u}_{L}, \mathbf{u}_{R}\right)$ will be proposed on the ground of a complete set of generalized jump relations, encoding the small scale effects taking place within viscous shock layers. The third section is devoted to the numerical approximation of solutions of the model with stiff viscous shocks for mesh refinements of practical interest. Such solutions are thus by definition underresolved. Classical splitting schemes built from suitable Godunov-type methods are shown to fail in satisfying the set of generalized jump conditions. The error analysis suggests then the introduction of a correction step, the so-called nonlinear projection procedure, to enforce for validity these generalized jump conditions. This new procedure achieves this requirement fairly independently of the mesh refinement. The desirable stability properties of relevant Godunov methods are shown to be preserved by this procedure. Finally in the last section, several numerical results are displayed, intending to illustrate the benefits of the proposed nonlinear projection.

\section{The mathematical model}

We consider a gas with density $\rho>0$ and velocity $u$ which is modelled by 2 general pressure laws $p_{1}\left(\tau, \epsilon_{1}\right)$ and $p_{2}\left(\tau, \epsilon_{2}\right)$, where $\tau=1 / \rho$ is the specific volume and $\epsilon_{j}>0(j=1,2)$ denote two independent specific internal energies. Each pressure law $p_{j}\left(\tau, \epsilon_{j}\right)$ is assumed to obey the second law of thermodynamics: namely, there exists a specific entropy $s_{j}\left(\tau, \epsilon_{j}\right), s_{j}: \mathbb{R}_{+} \times \mathbb{R}_{+} \rightarrow \mathbb{R}(j=1,2)$ satisfying for some temperature $T_{j}\left(\tau, \epsilon_{j}\right)>0$ :

$$
-T_{j} d s_{j}=d \epsilon_{j}+p_{j} d \tau, j=1,2,
$$

with the following properties:

$$
\text { the mapping }\left(\tau, \epsilon_{j}\right) \rightarrow s_{j}\left(\tau, \epsilon_{j}\right) \text { is strictly convex, }
$$

and

$$
\frac{\partial s_{j}}{\partial \tau}\left(\tau, \epsilon_{j}\right)=-\frac{p_{j}\left(\tau, \epsilon_{j}\right)}{T_{j}\left(\tau, \epsilon_{j}\right)}<0, \quad \frac{\partial s_{j}}{\partial \epsilon_{j}}\left(\tau, \epsilon_{j}\right)=-\frac{1}{T_{j}\left(\tau, \epsilon_{j}\right)}<0, \quad j=1,2 .
$$

Next, each of the specific entropies is asked without restriction to meet the following asymptotic conditions for any given fixed $\tau>0$ :

$$
\lim _{\epsilon_{j} \rightarrow 0+} s_{j}\left(\tau, \epsilon_{j}\right)=+\infty, \quad \lim _{\epsilon_{j} \rightarrow+\infty} s_{j}\left(\tau, \epsilon_{j}\right)=-\infty, \quad j=1,2 .
$$

To avoid unnecessary technical details, we consider pressure laws with the following standard nonlinearity property:

$$
\text { the function } \tau \rightarrow p_{j}\left(\tau, s_{j}\right), s_{j} \text { being fixed, is strictly convex, } j=1,2,
$$

where $\left(\tau, s_{1}, s_{2}\right)$ are chosen as admissible thermodynamic variables (see (2.3)).

The $4 \times 4$ system of PDEs that governs the fluid model under consideration is:

$$
\left\{\begin{array}{l}
\partial_{t} \rho+\partial_{x} \rho u=0 \\
\partial_{t} \rho u+\partial_{x}\left(\rho u^{2}+p_{1}\left(\tau, \epsilon_{1}\right)+p_{2}\left(\tau, \epsilon_{2}\right)\right)=\partial_{x}\left(\left(\mu_{1}+\mu_{2}\right) \partial_{x} u\right) \\
\partial_{t} \rho \epsilon_{j}+\partial_{x} \rho \epsilon_{j} u+p_{j}\left(\tau, \epsilon_{j}\right) \partial_{x} u=\mu_{j}\left(\partial_{x} u\right)^{2}, j=1,2
\end{array}\right.
$$

and is associated with the following natural phase space:

$$
\Omega_{\mathbf{u}}=\left\{\mathbf{u}:=\left(\rho, \rho u, \rho \epsilon_{1}, \rho \epsilon_{2}\right)^{T} \in \mathbb{R}^{4} / \rho>0, \rho u \in \mathbb{R}, \rho \epsilon_{j}>0, j=1,2\right\} .
$$


To shorten the notations, the system (2.6) is given the following condensed form:

$$
\partial_{t} \mathbf{u}+\mathcal{A}(\mathbf{u}) \partial_{x} \mathbf{u}=\mathcal{E}\left(\mathbf{u}, \partial_{x x}^{2} \mathbf{u}\right), \quad x \in \mathbb{R}, t>0 .
$$

This convection-diffusion system can be understood as an extension of the standard compressible Navier-Stokes equations when considering an additional PDE for governing an additional pressure. Owing to the classical thermodynamic assumptions (2.1) -(2.2), the first-order underlying system in (2.6) is easily seen to be hyperbolic over the phase space $\Omega_{\mathbf{u}}$ with eigenvalues $\lambda_{1}(\mathbf{u})=u-c(\mathbf{u}), \lambda_{2}(\mathbf{u})=\lambda_{3}(\mathbf{u})=u$ and $\lambda_{4}(\mathbf{u})=u+c(\mathbf{u})$. Here, the sound speed $c(\mathbf{u})$ follows from $c^{2}(\mathbf{u})=c_{1}^{2}(\mathbf{u})+c_{2}^{2}(\mathbf{u})$ with $c_{j}^{2}(\mathbf{u})=\partial_{\rho} p_{j}\left(\rho, s_{j}\right)_{s_{j}}>0$ (see (2.2)-(2.3)). Invoking the convexity property (2.5), the two extreme fields are seen to be genuinely nonlinear while the two intermediate ones are linearly degenerate. Considering the diffusive effects, we focus hereafter our attention on the interplay of the viscosity coefficients and we neglect the Fourier laws. Depending on the closure relations for the viscosities $\mu_{1}, \mu_{2}$ and the pressure laws $p_{1}, p_{2}$, several distinct models from physics enter the present framework. The reader is referred to the Introduction for examples motivated by complex compressible materials and to the work by Berthon [1 for a comprehensive analysis of compressible turbulence models.

In most of the present paper, the viscosity coefficients are assumed to be fixed positive constants for the sake of simplicity in the discussion. The reader is referred to section 3.2 .3 for the case of varying coefficients.

In close symmetry with the classical Navier-Stokes equations, the smooth solutions of system (2.6) obey the following additional nontrivial scalar equations:

Lemma 2.1. Smooth solutions $\mathbf{u}: \mathbb{R} \times \mathbb{R}_{+} \rightarrow \Omega_{\mathbf{u}}$ of (2.6) satisfy the following conservation law:

$$
\partial_{t} \rho E(\mathbf{u})+\partial_{x}\left(\rho E(\mathbf{u})+p_{1}\left(\tau, \epsilon_{1}\right)+p_{2}\left(\tau, \epsilon_{2}\right)\right) u=\partial_{x}\left(\left(\mu_{1}+\mu_{2}\right) u \partial_{x} u\right)
$$

where the total energy $\rho E$ is defined by

$$
\rho E(\mathbf{u})=\frac{(\rho u)^{2}}{2 \rho}+\rho \epsilon_{1}+\rho \epsilon_{2} .
$$

These smooth solutions satisfy in addition the following balance equations:

$$
\partial_{t} \rho s_{j}(\mathbf{u})+\partial_{x} \rho s_{j}(\mathbf{u}) u=-\frac{\mu_{j}}{T_{j}}\left(\partial_{x} u\right)^{2}, j=1,2 .
$$

Consequently, smooth solutions of (2.6) obey

$$
\mu_{2} T_{1}\left(\partial_{t} \rho s_{1}(\mathbf{u})+\partial_{x} \rho s_{1}(\mathbf{u}) u\right)-\mu_{1} T_{2}\left(\partial_{t} \rho s_{2}(\mathbf{u})+\partial_{x} \rho s_{2}(\mathbf{u}) u\right)=0 .
$$

The proof of this easy result is left to the reader but note in passing that either $\rho E, \rho s_{1}$ or $\rho s_{2}$ may serve as independent variables in place of the specific internal energies (see again (2.3)). At the expense of assuming that one of the two viscosities identically vanishes, say $\mu_{2}$, then the admissible change of variable $\left(\rho, \rho u, \rho E, \rho s_{2}\right)$ clearly gives rise from (2.9) and (2.11) with $j=2$ to a system in conservation form for governing the smooth solutions of (2.6). But, the two viscosity coefficients must generally be prescribed with strictly positive values according to physics. The key point is then that the three balance equations (2.9) and (2.11) can be actually proved to be the only nontrivial additional equations for smooth solutions [1] (up to some standard nonlinear transforms in $s_{1}$ and $s_{2}$ ). Despite several close relationships with the usual Navier-Stokes system, the very discrepancy stays in the lack of four nontrivial conservation laws for governing four independent variables. 

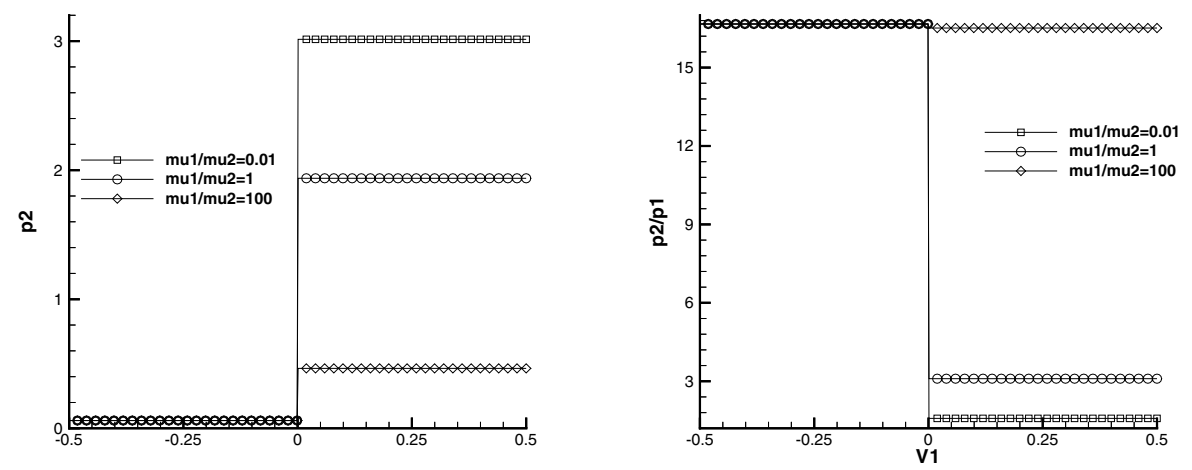

FIGURE 2. Right end state $\mathbf{u}_{R}$ as a function of $\mu_{1} / \mu_{2}$ for a given $\left(\sigma, \mathbf{u}_{L}\right)$

Without restrictive modelling assumptions (see below), none of the equations (2.11) and (2.12) boils down to a conservation law. As a consequence, the PDE model (2.6) cannot recast, generally speaking, in full conservation form.

Our purpose here is to highlight after the pioneering works by LeFloch [17, Raviart-Sainsaulieu 21 and Sainsaulieu 23] that the nonconservation form met by (2.6) makes the end states of viscous shock layers to intrinsically depend on the shape of the diffusive operator: here namely on the ratio of the viscosity coefficients $\mu_{1}$ and $\mu_{2}$. In order to assess this issue, let us focus our attention on the nonstandard balance equation (2.12) when temporarily adopting the next restrictive modeling assumptions on the viscosity coefficients.

Lemma 2.2. Let $\mu_{1}^{0}$ and $\mu_{2}^{0}$ denote two positive constants and assume that $\mu_{1}=$ $\mu_{1}^{0} T_{1}, \mu_{2}=\mu_{2}^{0} T_{2}$. Then (2.12) coincides with the following conservation law:

$$
\partial_{t} \rho\left(\mu_{2}^{0} s_{1}-\mu_{1}^{0} s_{2}\right)+\partial_{x} \rho\left(\mu_{2}^{0} s_{1}-\mu_{1}^{0} s_{2}\right) u=0 .
$$

The above assumptions therefore allow for an additional nontrivial conservation law encoded in the nonstandard balance equation (2.12). The associated RankineHugoniot condition clearly indicates that the end states of the viscous shock layers under consideration actually depend on the ratio $\mu_{2}^{0} / \mu_{1}^{0}$. The dependence we have just pointed out is numerically illustrated in Figure 2 in a more general setting in which the system (2.6) does not admit an equivalent full conservation form. For a given left end state $\mathbf{u}_{L}$ and a given velocity $\sigma$, the required right end states $\mathbf{u}_{R}$ are defined when solving numerically the nonlinear ODEs system governing travelling wave solutions (see next section) for various ratios of the viscosities. Furthermore, (2.12) continues to play a major role in a general setting since it encodes a generalized jump condition which turns out to play a central role for our numerical purpose.

2.1. Travelling wave solutions and jump relations. In this section, we derive generalized jump relations that are needed to characterize the triple $\left(\sigma ; \mathbf{u}_{L}, \mathbf{u}_{R}\right)$ associated with a given viscous shock layer. To that end, let us first recall that a travelling wave solution of (2.6) is a smooth solution in the form $\mathbf{u}(x, t)=\widehat{\mathbf{u}}(x-\sigma t)$, 
$\sigma \in \mathbb{R}$, with

$$
\lim _{\xi \rightarrow-\infty} \widehat{\mathbf{u}}(\xi)=\mathbf{u}_{L}, \quad \lim _{\xi \rightarrow+\infty} \widehat{\mathbf{u}}(\xi)=\mathbf{u}_{R}, \quad \xi=x-\sigma t,
$$

where $\left(\mathbf{u}_{L}, \mathbf{u}_{R}\right) \in \Omega_{\mathbf{u}}^{2}$. Besides (2.14),$\widehat{\mathbf{u}}(\xi)$ must satisfy the following nonlinear ODE system:

$$
-\sigma d_{\xi} \widehat{\mathbf{u}}+\mathcal{A}(\widehat{\mathbf{u}}) d_{\xi} \widehat{\mathbf{u}}=\mathcal{E}\left(\widehat{\mathbf{u}}, d_{\xi \xi}^{2} \widehat{\mathbf{u}}\right), \quad \xi \in \mathbb{R},
$$

where we have used the condensed form (2.8) of system (2.6). Berthon and Coquel 2 have proved global existence and uniqueness of smooth travelling wave solutions of (2.6) for fairly general (nonlinear) viscosity functions and pressure laws.

Let us then observe after LeFloch [17, Raviart-Sainsaulieu [21, that the end states of the integral curve solution of (2.14), (2.15) do not depend on the amplitude of the diffusion tensor modelled in (2.15) but just on its shape. To that end, let us introduce the function

$$
\mathbf{u}_{\delta}(x, t)=\widehat{\mathbf{u}}\left(\frac{x-\sigma t}{\delta}\right),
$$

where $\delta>0$ denotes a positive rescaling parameter. For any given fixed $\delta>0$, $\mathbf{u}_{\delta}(x, t)$ turns out to be a travelling wave solution of (2.6) but for the rescaled viscosities $\mu_{1}^{\delta}$ and $\mu_{2}^{\delta}$ defined by

$$
\mu_{1}^{\delta}=\delta \mu_{1}, \quad \mu_{2}^{\delta}=\delta \mu_{2} .
$$

Here, $\delta$ plays nothing but the role of the inverse Reynolds number. The travelling wave $\mathbf{u}_{\delta}(x, t)$ obviously achieves the same endpoints $\mathbf{u}_{L}$ and $\mathbf{u}_{R}$ and propagates with speed $\sigma$.

As a consequence, the following results hold true (see [3] for a proof and extensions to a more general setting):

Theorem 2.3. Assume that $\mu_{1}$ and $\mu_{2}$ denote positive constants. Then the triple $\left(\sigma, \mathbf{u}_{L}, \mathbf{u}_{R}\right)$ associated with the resulting dissipative tensor necessarily obeys the jump relations:

$$
\left\{\begin{array}{l}
-\sigma[\rho]+[\rho u]=0, \\
-\sigma[\rho u]+\left[\rho u^{2}+p_{1}+p_{2}\right]=0, \\
-\sigma[\rho E]+\left[\left(\rho E+p_{1}+p_{2}\right) u\right]=0,
\end{array}\right.
$$

and necessarily satisfies the two entropy inequalities:

$$
\left\{\begin{array}{l}
-\sigma\left[\rho s_{1}\right]+\left[\rho s_{1} u\right]<0 \\
-\sigma\left[\rho s_{2}\right]+\left[\rho s_{2} u\right]<0
\end{array}\right.
$$

In order to specify (2.18), let us consider for any given $\delta>0, \mathbf{u}_{\delta}$ a rescaled travelling wave. Let us then define from (2.16) and with clear notation:

$$
\begin{aligned}
& \int_{\mathbb{R}}\left(\widehat{T_{1}}\right)_{\delta}\left(\partial_{t}\left(\widehat{\rho s_{1}}\right)_{\delta}+\partial_{x}\left(\widehat{\rho s_{1} u}\right)_{\delta}\right)(\xi) d \xi \\
& \quad=-\delta \mu_{1} \int_{\mathbb{R}}\left(d_{\xi} u\right)^{2} d \xi=-\mu_{1} \int_{\mathbb{R}}\left(d_{\xi} u\right)^{2} d \xi \equiv \zeta_{1}\left(\sigma ; \mathbf{u}_{L}, \mathbf{u}_{R}\right), \\
& \int_{\mathbb{R}}\left(\widehat{T_{2}}\right)_{\delta}\left(\partial_{t}\left(\widehat{\rho s_{2}}\right)_{\delta}+\partial_{x}\left(\widehat{\rho s_{2} u}\right)_{\delta}\right)(\xi) d \xi=\zeta_{2}\left(\sigma ; \mathbf{u}_{L}, \mathbf{u}_{R}\right) .
\end{aligned}
$$


Then the dissipation rates $\zeta_{1}$ and $\zeta_{2}$ entering the above two entropy inequalities are bounded and do not depend on the rescaling parameter $\delta>0$ but only depend on the ratio of the viscosities through the following identity:

$$
\mu_{2} \zeta_{1}\left(\sigma ; \mathbf{u}_{L}, \mathbf{u}_{R}\right)-\mu_{1} \zeta_{2}\left(\sigma ; \mathbf{u}_{L}, \mathbf{u}_{R}\right)=0 .
$$

Rephrasing the above result, $\left(\sigma ; \mathbf{u}_{L}, \mathbf{u}_{R}\right)$ is entirely characterized by the classical jump relations (2.17) and the generalized jump condition (2.21). Despite that (2.21) cannot be given, generally speaking, a closed form of expression, it seems convenient to re-express it equivalently when introducing two suitable averaged temperatures as follows:

$$
\begin{aligned}
& \overline{T_{1}}\left\{-\sigma\left[\rho s_{1}\right]+\left[\rho s_{1} u\right]\right\}=\zeta_{1}\left(\sigma ; \mathbf{u}_{L}, \mathbf{u}_{R}\right) \\
& \overline{T_{2}}\left\{-\sigma\left[\rho s_{2}\right]+\left[\rho s_{2} u\right]\right\}=\zeta_{2}\left(\sigma ; \mathbf{u}_{L}, \mathbf{u}_{R}\right)
\end{aligned}
$$

so that (2.21) now reads:

$$
\mu_{2} \overline{T_{1}}\left\{-\sigma\left[\rho s_{1}\right]+\left[\rho s_{1} u\right]\right\}-\mu_{1} \overline{T_{2}}\left\{-\sigma\left[\rho s_{2}\right]+\left[\rho s_{2} u\right]\right\}=0 .
$$

This form of the generalized jump condition obviously finds its roots in the companion PDE (2.12).

Remark 2.4. In view of the jump relations (2.17) and (2.22), one of the two thermodynamic entropies, either $\rho s_{1}$ or $\rho s_{2}$, must obviously be understood as a nonlinear function of the four remaining independent variables; respectively $\rho s_{1}:=$ $\left\{\rho s_{1}\right\}\left(\rho, \rho u, \rho E, \rho s_{2}\right)$ or $\rho s_{2}:=\left\{\rho s_{2}\right\}\left(\rho, \rho u, \rho E, \rho s_{1}\right)$.

Notice after [17] and [21] that the family of travelling wave solutions $\{\hat{\mathbf{u}}\}_{\delta>0}$, for a given triple $\left(\sigma ; \mathbf{u}_{L}, \mathbf{u}_{R}\right)$, can be seen to converge as $\delta$ goes to zero in the $L_{l o c}^{1}$ strong topology to the following step function:

$$
\mathbf{u}_{0}(x, t)= \begin{cases}\mathbf{u}_{L}, & x<\sigma t \\ \mathbf{u}_{R}, & x>\sigma t .\end{cases}
$$

Let us underline that this so-called shock-solution satisfies by construction the (generalized) jump conditions (2.17) and (2.22). Note from (2.16) that numerically speaking, capturing viscous shock layers in the regime of large Reynolds, i.e. for vanishing $\delta$, means nothing but capturing the underlying shock solution (2.23).

2.2. Equivalent formulations and convexity properties. Here the extended Navier-Stokes system (2.6) is given two equivalent formulations for smooth solutions that allow for highly desirable convexity properties in the analysis of the forthcoming numerical methods. Indeed the feasibility of a stable method for properly capturing the sharp travelling wave solutions of (2.6) in the regime of large Reynolds numbers will essentially result from convexity arguments. The convexity properties of central importance in the sequel are stated right now: 
Lemma 2.5. Under the thermodynamic assumptions (2.1)-(2.3), the mappings $\left(\tau, s_{j}\right) \rightarrow \epsilon_{j}\left(\tau, s_{j}\right)$ are well defined and strictly convex. In addition, the mappings

$$
\begin{aligned}
& \left(\rho, \rho u, \rho E, \rho s_{2}\right) \rightarrow\left\{\rho s_{1}\right\}\left(\frac{1}{\rho}, \frac{\rho E-\frac{(\rho u)^{2}}{2 \rho}-\rho \epsilon_{2}\left(\frac{1}{\rho}, \frac{\rho s_{2}}{\rho}\right)}{\rho}\right), \\
& \left(\rho, \rho u, \rho E, \rho s_{1}\right) \rightarrow\left\{\rho s_{2}\right\}\left(\frac{1}{\rho}, \frac{\rho E-\frac{(\rho u)^{2}}{2 \rho}-\rho \epsilon_{1}\left(\frac{1}{\rho}, \frac{\rho s_{1}}{\rho}\right)}{\rho}\right)
\end{aligned}
$$

are strictly convex.

The proof is postponed to the end of the present section.

To take advantage of these convexity results, the first equivalent system for smooth solutions that we consider is obtained when qualifying $\mathbf{v}=\left(\rho, \rho u, \rho E, \rho s_{2}\right)^{T}$ as the unknown and it is thus written as:

$$
\left\{\begin{array}{l}
\partial_{t} \rho+\partial_{x} \rho u=0, \quad x \in \mathbb{R}, t>0 \\
\partial_{t} \rho u+\partial_{x}\left(\rho u^{2}+p_{1}+p_{2}\right)=\partial_{x}\left(\left(\mu_{1}+\mu_{2}\right) \partial_{x} u\right) \\
\partial_{t} \rho E+\partial_{x}\left(\rho E+p_{1}+p_{2}\right) u=\partial_{x}\left(\left(\mu_{1}+\mu_{2}\right) u \partial_{x} u\right) \\
\partial_{t} \rho s_{2}+\partial_{x} \rho s_{2} u=-\frac{\mu_{2}}{T_{2}}\left(\partial_{x} u\right)^{2}
\end{array}\right.
$$

The associated natural phase space reads:

$$
\Omega_{\mathbf{v}}=\left\{\mathbf{v} \in \mathbb{R}^{4} ; \rho>0, \rho u \in \mathbb{R}, \rho E-\frac{(\rho u)^{2}}{2 \rho}-\rho \epsilon_{2}\left(\frac{1}{\rho}, \frac{\rho s_{2}}{\rho}\right)>0, \rho s_{2} \in \mathbb{R}\right\}
$$

Here the pair $\left(\rho s_{1}, \rho s_{1} u\right): \Omega_{\mathbf{v}} \times \Omega_{\mathbf{v}} \rightarrow R^{2}$ with $\mathbf{v} \rightarrow\left\{\rho s_{1}\right\}(\mathbf{v})$ strictly convex can be understood as a Lax entropy pair but for which smooth solutions of (2.26) must satisfy without additional conditions the following scalar law with a prescribed rate of entropy dissipation (see Lemma 2.1):

$$
\partial_{t}\left\{\rho s_{1}\right\}(\mathbf{v})+\partial_{x}\left\{\rho s_{1} u\right\}(\mathbf{v})=-\frac{\mu_{1}}{T_{1}}\left(\partial_{x} u\right)^{2} .
$$

Some desirable stability properties will be inferred from (2.28) (thus symmetrically from (2.26) despite that $\rho s_{2}$ and $\left\{\rho s_{1}\right\}(\mathbf{v})$ do not, strictly speaking, play the same role), just after the description of the second equivalent system that we now want to promote. The key balance equation (2.12) is explicitly involved in this, so as to consider:

$$
\begin{aligned}
& \partial_{t} \rho+\partial_{x} \rho u=0, \quad x \in \mathbb{R}, t>0, \\
& \partial_{t} \rho u+\partial_{x}\left(\rho u^{2}+p_{1}+p_{2}\right)=\partial_{x}\left(\left(\mu_{1}+\mu_{2}\right) \partial_{x} u\right), \\
& \partial_{t} \rho E+\partial_{x}\left(\rho E+p_{1}+p_{2}\right) u=\partial_{x}\left(\left(\mu_{1}+\mu_{2}\right) u \partial_{x} u\right), \\
& \mu_{1} T_{2}\left\{\partial_{t} \rho s_{2}+\partial_{x} \rho s_{2} u\right\}-\mu_{2} T_{1}\left\{\partial_{t} \rho s_{1}+\partial_{x} \rho s_{1} u\right\}=0,
\end{aligned}
$$

so that $\rho s_{1}$ and $\rho s_{2}$ are kept in symmetry. The very motivation for this nonclassical formulation obviously stems from the fact that (2.29d) is intrinsically associated with the generalized jump relation (2.22). In this respect, (2.29) will play a central role in the forthcoming numerical method. Since the above system involves five partial derivatives in time for only four independent unknowns, either the total energy $\rho E$ or one of the two specific entropies, $\rho s_{1}$ or $\rho s_{2}$, must be understood as a nonlinear function of the four remaining independent variables: namely either $\rho E:=\{\rho E\}\left(\rho, \rho u, \rho s_{1}, \rho s_{2}\right)$ or $\rho s_{1}:=\left\{\rho s_{1}\right\}\left(\rho, \rho u, \rho E, \rho s_{2}\right)$, or 
$\rho s_{2}:=\left\{\rho s_{2}\right\}\left(\rho, \rho u, \rho E, \rho s_{1}\right)$. To fix the ideas, let $\mathbf{v}=\left(\rho, \rho u, \rho E, \rho s_{2}\right)$ play without restriction the role of the unknown. The underlying first-order system in (2.29) then takes the somewhat unusual form:

$$
\begin{aligned}
& \partial_{t} \rho+\partial_{x} \rho u=0, \quad x \in \mathbb{R}, t>0, \\
& \partial_{t} \rho u+\partial_{x}\left(\rho u^{2}+p_{1}+p_{2}\right)=0, \\
& \partial_{t} \rho E+\partial_{x}\left(\rho E+p_{1}+p_{2}\right) u=0, \\
& \mu_{1} T_{2}(\mathbf{v})\left\{\partial_{t} \rho s_{2}+\partial_{x} \rho s_{2} u\right\}-\mu_{2} T_{1}(\mathbf{v})\left\{\partial_{t}\left\{\rho s_{1}\right\}(\mathbf{v})+\partial_{x}\left\{\rho s_{1} u\right\}(\mathbf{v})\right\}=0,
\end{aligned}
$$

but actually enters the basic theory for nonlinear hyperbolic systems. Indeed, the linearization of (2.30) at any given constant state $\mathbf{v}_{0} \in \Omega_{\mathbf{v}}$ reads with clear notation:

$$
\mathcal{A}\left(\mathbf{v}_{0}\right) \partial_{t} \mathbf{v}+\mathcal{B}\left(\mathbf{v}_{0}\right) \partial_{x} \mathbf{v}=0
$$

where direct calculations show that $\operatorname{det}\left(\mathcal{A}\left(\mathbf{v}_{0}\right)\right)=\mu_{1} T_{2}^{0}-\mu_{2} T_{1}^{0} \partial_{\rho s_{2}}\left\{\rho s_{1}\right\}\left(\mathbf{v}_{\mathbf{0}}\right)=$ $\left(\mu_{1}+\mu_{2}\right) T_{2}^{0}>0$. Hence, $\mathcal{A}\left(\mathbf{v}_{0}\right)$ is invertible for any given $\mathbf{v}_{0} \in \Omega_{\mathbf{v}}$ and the usual $L^{2}$ stability analysis applies: of course, (2.30) is a nonlinear hyperbolic system but in nonconservation form (see LeFloch [17] for other examples). The next statement yields in particular the expected equivalence of (2.29) and (2.26) but also gives rise to a priori estimates of importance in the sequel:

Lemma 2.6. Smooth solutions $\mathbf{v}: \mathbb{R} \times \mathbb{R}_{+} \rightarrow \Omega_{\mathbf{v}}$ of (2.29) obey the following Lax entropy balance equations with a prescribed rate of entropy dissipation:

$$
\partial_{t}\left\{\rho s_{j}\right\}(\mathbf{v})+\partial_{x}\left\{\rho s_{j} u\right\}(\mathbf{v})=-\frac{\mu_{j}}{T_{j}}\left(\partial_{x} u\right)^{2}, \quad j=1,2 .
$$

In addition, the next entropy-like inequalities hold true for any given smooth increasing function $\phi_{j}: \mathbb{R} \rightarrow \mathbb{R}_{+}$:

$$
\partial_{t}\left\{\rho \phi_{j}\left(s_{j}\right)\right\}(\mathbf{v})+\partial_{x}\left\{\rho \phi_{j}\left(s_{j}\right) u\right\}(\mathbf{v}) \leq 0, \quad j=1,2 .
$$

The interest in the inequalities (2.32) stems from the resulting formal a priori estimates:

$$
0 \leq \int_{\mathbb{R}}\left\{\rho \phi_{j}\left(s_{j}\right)\right\} \mathbf{v}(x, t) d x \leq \int_{\mathbb{R}}\left\{\rho \phi_{j}\left(s_{j}\right)\right\} \mathbf{v}_{0}(x) d x,
$$

where $\mathbf{v}_{0}: \mathbb{R} \rightarrow \Omega_{\mathbf{v}}$ denotes some initial data for the Cauchy problem (2.29) (or equivalently (2.26) ). Using related estimates, Tadmor [25] has proved the next important maximum principle on the specific entropies (see [25] for a refined version):

$$
s_{j}(\mathbf{v}(x, t)) \leq \max _{y \in \mathbb{R}}\left(s_{j}\left(\mathbf{v}_{0}(y)\right)\right), \quad j=1,2, \quad \text { a.e. } x \in \mathbb{R}, t>0,
$$

when privileging $\phi_{j}\left(s_{j}\right)=\max \left(0, s_{j}-\max _{y \in \mathbb{R}}\left(s_{j}\left(\mathbf{v}_{0}(y)\right)\right)\right.$. Most of the inequalities (2.32) and in particular their consequence (2.34) will be recovered in the numerical method we develop hereafter. These desirable stability estimates will in particular allow us to prove that the discrete method preserves the positivity of the internal energies $\epsilon_{j}, j=1,2$.

Proof. The equations (2.29a)-(2.29c) can be seen to yield from the second law of thermodynamics (2.1) the next identity:

$$
-T_{1}\left\{\partial_{t} \rho s_{1}+\partial_{x} \rho s_{1} u\right\}-T_{2}\left\{\partial_{t} \rho s_{2}+\partial_{x} \rho s_{2} u\right\}=\left(\mu_{1}+\mu_{2}\right)\left(\partial_{x} u\right)^{2},
$$


and (2.31) follows when invoking (2.29d). Inequalities (2.32) are then recovered from

$$
\rho\left\{\partial_{t} \phi_{j}\left(s_{j}\right)+u \partial_{x} \phi_{j}\left(s_{j}\right)\right\}=-\frac{\mu_{j} \phi_{j}^{\prime}\left(s_{j}\right)}{T_{j}}\left(\partial_{x} u\right)^{2} \leq 0, \quad j=1,2 .
$$

Let us conclude the present section by establishing the convexity Lemma 2.5. The proof readily follows from the technical results given below. The following first statement is proved in Godlewski-Raviart [1], chapter 2.

Lemma 2.7. Let the index $j \in\{1,2\}$ be fixed. Under the thermodynamic assumptions (2.2)-2.3), the following assertions are equivalent:

(i) The mapping $\left(\tau, \epsilon_{j}\right) \rightarrow s_{j}\left(\tau, \epsilon_{j}\right)$ is strictly convex.

(ii) The mapping $\left(\tau, s_{j}\right) \rightarrow \epsilon_{j}\left(\tau, s_{j}\right)$ is strictly convex.

(iii) The mapping $\left(\rho, \rho s_{j}\right) \rightarrow \rho \epsilon_{j}\left(\frac{1}{\rho}, \frac{\rho s_{j}}{\rho}\right)$ is strictly convex.

This classical result is easily seen to imply:

Lemma 2.8. The mapping

$$
\left(\rho, \rho u, \rho s_{1}, \rho s_{2}\right) \rightarrow \frac{(\rho u)^{2}}{2 \rho}+\rho \epsilon_{1}\left(\frac{1}{\rho}, \frac{\rho s_{1}}{\rho}\right)+\rho \epsilon_{2}\left(\frac{1}{\rho}, \frac{\rho s_{2}}{\rho}\right)
$$

is strictly convex.

Equipped with these results, we are now in a position to establish the convexity Lemma 2.5.

Proof of Lemma 2.5. Let us restrict our attention to the mapping $\mathbf{v} \rightarrow\left\{\rho s_{1}\right\}(\mathbf{v})$ since the companion entropy $\left\{\rho s_{2}\right\}$ plays a symmetric role. The strict convexity of the mapping $\mathbf{w}:=\left(\rho, \rho u, \rho s_{1}, \rho s_{2}\right) \rightarrow\{\rho E\}(\mathbf{w})$ (see Lemma 2.8) is known to be equivalent to the validity of the next inequality for any given pair $\left(\mathbf{w}_{L}, \mathbf{w}_{R}\right) \in \Omega_{\mathbf{w}}^{2}$ :

$$
\{\rho E\}\left(\mathbf{w}_{R}\right)-\{\rho E\}\left(\mathbf{w}_{L}\right)>\nabla_{\mathbf{w}}\{\rho E\}\left(\mathbf{w}_{L}\right) \cdot\left(\mathbf{w}_{R}-\mathbf{w}_{L}\right) .
$$

This one is seen to recast as follows:

$$
\begin{aligned}
& -\partial_{\rho s_{1}}\{\rho E\}\left(\mathbf{w}_{L}\right)\left(\left(\rho s_{1}\right)_{R}-\left(\rho s_{1}\right)_{L}\right) \\
& >\partial_{\rho}\{\rho E\}\left(\mathbf{w}_{L}\right)\left(\rho_{R}-\rho_{L}\right)+\partial_{\rho u}\{\rho E\}\left(\mathbf{w}_{L}\right)\left((\rho u)_{R}-(\rho u)_{L}\right) \\
& +\partial_{\rho s_{2}}\{\rho E\}\left(\mathbf{w}_{L}\right)\left(\left(\rho s_{2}\right)_{R}-\left(\rho s_{2}\right)_{L}\right)-\left(\{\rho E\}\left(\mathbf{w}_{R}\right)-\{\rho E\}\left(\mathbf{w}_{L}\right)\right) .
\end{aligned}
$$

Since $\partial_{\rho s_{1}}\{\rho E\}(\mathbf{w})=\partial_{s_{1}} \epsilon_{1}\left(\tau, s_{1}\right)=-T_{1}<0$ in view of (2.3) , the inverse mapping $\mathbf{v}=\left(\rho, \rho u, \rho E, \rho s_{2}\right)^{T} \rightarrow\left\{\rho s_{1}\right\}(\mathbf{v})$ is well defined and the classical chain rule gives

$$
\partial_{\rho E}\left\{\rho s_{1}\right\}(\mathbf{v})=\frac{1}{\partial_{\rho s_{1}}\{\rho E\}}(\mathbf{w}(\mathbf{v})), \quad \partial_{X}\left\{\rho s_{1}\right\}(\mathbf{v})=-\frac{\partial_{X}\{\rho E\}}{\partial_{\rho s_{1}}\{\rho E\}}(\mathbf{w}(\mathbf{v})),
$$

where $X$ denotes either $\rho, \rho u$ or $\rho s_{2}$. Since $-\partial_{\rho s_{1}}\{\rho E\}(\mathbf{w})>0$, we thus arrive from (2.36) at the following inequality, valid for all pairs $\left(\mathbf{v}_{L}, \mathbf{v}_{R}\right) \in \Omega_{\mathbf{v}}^{2}$ :

$$
\left\{\rho s_{1}\right\}\left(\mathbf{v}_{R}\right)-\left\{\rho s_{1}\right\}\left(\mathbf{v}_{L}\right)>\nabla_{\mathbf{v}}\left\{\rho s_{1}\right\}\left(\mathbf{v}_{L}\right) \cdot\left(\mathbf{v}_{R}-\mathbf{v}_{L}\right)
$$

which is nothing but the required result. 


\section{Godunov Methods With NONLINEAR PROJECTIONS}

This section is devoted to the numerical approximation of the solutions of the convection-diffusion system (2.6) in the regime of a large Reynolds number with a special emphasis put on the satisfaction of the generalized jump condition (2.22) at the discrete level. As pointed out in the previous sections, several equivalent formulations of (2.6) do coexist but, of course, they are all associated with the same generalized jump conditions.

As proved below, such an equivalence property, valid for exact solutions, turns out to be lost in general at the discrete level within the framework of classical numerical methods: namely methods built from a consistent operator splitting into the underlying hyperbolic system and its viscous perturbation. For instance, such seemingly well-grounded methods when derived from the formulation (2.26), i.e in the unknown $\mathbf{v}=\left(\rho, \rho u, \rho E, \rho s_{2}\right)$, and next from the companion form expressed in the $\left(\rho, \rho u, \rho E, \rho s_{1}\right)$ state variable, systematically produce grossly different approximate solutions. These in addition exhibit unacceptable errors with the exact solution. As established below, errors in the usual approach essentially find their roots in the numerical approximation of the underlying hyperbolic system. Roughly speaking, if suitable finite difference formulae for approximating the second-order operator can actually be devised so as to preserve the generalized jump conditions in (2.29), the use of stable approximate Riemann solvers inevitably results in a violation of these relations. The core of this breakdown rests with the averaging procedure of neighbouring approximate Riemann solutions over each computational cell. Essentially because of the Jensen inequality, the $L^{2}$-averaging induces a too large amount of artificial entropy dissipation, which overtakes the one prescribed in the exact PDEs. Exemplifying this assertion, the Lax entropy $\left\{\rho s_{1}\right\}(\mathbf{v})$ turns out to be over-dissipated when $\mathbf{v}=\left(\rho, \rho u, \rho E, \rho s_{2}\right)$ serves as the unknown and so does conversely $\left\{\rho s_{2}\right\}(\mathbf{w})$ when invoking $\mathbf{w}=\left(\rho, \rho u, \rho E, \rho s_{1}\right)$ as the state variable. In both cases, $\rho s_{1}(x, t)$ and $\rho s_{2}(x, t)$ cannot be kept in balance according to (2.12). This flaw just translates into a violation of the required generalized jump condition (2.22) coming with (2.12), and we again refer the reader to the results displayed in Figure 1 for an illustration of the heavily negative consequences.

To bypass this failure, we introduce below a nonlinear projection procedure. Involved as an additional and last step to any given relevant splitting method, this procedure provides a simple and effective correction in enforcing the two entropies $\rho s_{1}$ and $\rho s_{2}$ to be kept in balance in their evolution in time according to (2.12). As strongly attested by the numerical experiments, discrete solutions obtained by the nonlinear projection procedure achieve a far better agreement with exact solutions.

The key issue in the feasibility of the nonlinear correction step (existence and uniqueness of a solution of a scalar nonlinear equation in each cell) surprisingly stays in the property that one of the two entropies, namely the Lax entropy, has been over-dissipated in the splitting step. In other words, the correction can only take place provided that a stable (and thus dissipative) approximate Riemann solver has been involved in the prediction step. In addition, the nonlinear correction procedure is proved to inherit all the stability properties from the underlying classical approximate Riemann solver: namely positivity properties, a full set of discrete entropy inequalities and a maximum principle for the specific entropies.

The format of this section is dictated by the steps entering the predictioncorrection method that we propose for approximating the solutions of system (2.29). 
First, a convenient splitting method is derived on the ground of the equivalent form (2.26) to serve as a building block in the prediction step. Its main properties are briefly discussed with central attention paid to the violation of the key balance equation (2.29d). Then the nonlinear projection procedure is introduced and the stability properties met by this correction step are analysed.

3.1. Godunov-type methods and $L^{2}$ projections. This prediction step makes use of the equivalent form (2.26); i.e., we privilege $\mathbf{v}=\left(\rho, \rho u, \rho E, \rho s_{2}\right)^{T}$ to be the state variable, so as to take advantage of the convexity property (2.24) stated in Lemma 2.5. Let $\Delta t$ and $\Delta x$ respectively denote the time and space increments, chosen to be constant for simplicity. The numerical approximate solution, $\mathbf{v}_{\Delta x}$ : $\mathbb{R} \times \mathbb{R}_{+} \rightarrow \Omega_{\mathbf{v}}$, is as usual supposed to be piecewise constant, and we set using classical notation:

$$
\mathbf{v}_{\Delta x}(x, t)=\mathbf{v}_{i}^{n}, \quad(x, t) \in\left(x_{i-1 / 2}, x_{i+1 / 2}\right) \times\left(t^{n}, t^{n+1}\right), \quad i \in \mathbb{Z}, n \in \mathbb{N} .
$$

3.1.1. First step: Underlying first-order system $\left(t^{n} \rightarrow t^{n+1,=}\right)$. Assume that the discrete solution $\mathbf{v}_{\Delta x}\left(x, t^{n}\right)$ is known at the time level $t^{n}$. According to the standard splitting strategy, this one is first partially updated when solving for small times $t \in(0, \Delta t)$ the following Cauchy problem:

$$
\left\{\begin{array}{l}
\partial_{t} \rho+\partial_{x} \rho u=0, \quad x \in \mathbb{R}, t>0, \\
\partial_{t} \rho u+\partial_{x}\left(\rho u^{2}+p_{1}+p_{2}\right)=0, \\
\partial_{t} \rho E+\partial_{x}\left(\rho E+p_{1}+p_{2}\right) u=0, \\
\partial_{t} \rho s_{2}+\partial_{x} \rho s_{2} u=0,
\end{array}\right.
$$

with $\mathbf{v}_{h}\left(x, t^{n}\right)$ as initial data. For the sake of uniqueness, weak solutions of the above nonlinear hyperbolic system are classically selected according to the Lax entropy inequality (see Lemma 2.5):

$$
\partial_{t}\left\{\rho s_{1}\right\}(\mathbf{v})+\partial_{x}\left\{\rho s_{1} u\right\}(\mathbf{v}) \leq 0 .
$$

For convenience in the notation, the system (3.2) is given the clear condensed form:

$$
\partial_{t} \mathbf{v}+\partial_{x} \mathbf{g}(\mathbf{v})=0 .
$$

Under the CFL-like restriction:

$$
\frac{\Delta t}{\Delta x} \max _{\mathbf{v}}\left|\lambda_{i}(\mathbf{v})\right| \leq \frac{1}{2}
$$

to be met for all the $\mathbf{v}$ under consideration, the exact solution of (3.2)-(3.3) is classically made of the superposition of the sequence of noninteracting Riemann solutions emerging at each interface $x_{i+1 / 2}$, which we denote by $\omega\left(. ; \mathbf{v}_{i}^{n}, \mathbf{v}_{i+1}^{n}\right)$. This exact solution is then averaged over each cell to yield the following piecewise constant partial update:

$$
\mathbf{v}_{i}^{n+1,=}=\mathbf{v}_{i}^{n}-\frac{\Delta t}{\Delta x}\left\{\mathbf{g}\left(\omega\left(0^{+} ; \mathbf{v}_{i}^{n}, \mathbf{v}_{i+1}^{n}\right)\right)-\mathbf{g}\left(\omega\left(0^{+} ; \mathbf{v}_{i-1}^{n}, \mathbf{v}_{i}^{n}\right)\right)\right\}, \quad i \in \mathbb{Z} .
$$

Next, in view of the convexity Lemma (2.5), classical considerations (see for instance [11) ensure that under the CFL restriction (3.5), the update $\left(\rho s_{1}\right)_{i}^{n+1,=} \equiv$ $\left\{\rho s_{1}\right\}\left(\mathbf{v}_{i}^{n+1,=}\right)$ obeys as a consequence of the $L^{2}$-averaging procedure in (3.6) :

$$
\begin{aligned}
\left(\rho s_{1}\right)(\mathbf{v})_{i}^{n+1,=}-\left(\rho s_{1}\right)_{i}^{n}+\frac{\Delta t}{\Delta x}\{ & \left\{\rho s_{1}\right\}\left(\omega\left(0^{+} ; \mathbf{v}_{i}^{n}, \mathbf{v}_{i+1}^{n}\right)\right. \\
& \left.-\left\{\rho s_{1}\right\}\left(\omega\left(0^{+} ; \mathbf{v}_{i-1}^{n}, \mathbf{v}_{i}^{n}\right)\right)\right\} \leq 0 .
\end{aligned}
$$


This inequality is, in general, strict because of the entropy satisfying shock solutions in (3.2) -(3.3) but also in view of the well-known Jensen inequality (see 11] for the details):

$$
\begin{aligned}
& \left\{\rho s_{1}\right\}\left(\mathbf{v}_{i}^{n+1,=}\right)=\frac{1}{\Delta x} \int_{x_{i-1 / 2}}^{x_{i+1 / 2}}\left\{\rho s_{1}\right\}\left(\rho, \rho u, \rho E, \rho s_{2}\right)\left(x, t^{n+1,=}\right) d x \\
& -\frac{1}{\Delta x} \int_{x_{i-1 / 2}}^{x_{i+1 / 2}}\left(\left(\mathbf{v}\left(x, t^{n+1,=}\right)-\mathbf{v}_{i}^{n+1,=}\right),\left\{\overline{\nabla^{2} \rho s_{1}}\right\}_{i}^{n+1,=}\left(\mathbf{v}\left(x, t^{n+1,=}\right)-\mathbf{v}_{i}^{n+1,=}\right)\right) d x,
\end{aligned}
$$

where we have set

$$
\left\{\overline{\nabla^{2} \rho s_{1}}\right\}_{i}^{n+1,=}=\int_{0}^{1}\left\{\nabla^{2} \rho s_{1}\right\}\left(s \mathbf{v}\left(x, t^{n+1,=}\right)+(1-s) \mathbf{v}_{i}^{n+1,=}\right) s(1-s) d s
$$

to obtain

$$
\left\{\rho s_{1}\right\}\left(\mathbf{v}_{i}^{n+1,=}\right) \leq \frac{1}{\Delta x} \int_{x_{i-1 / 2}}^{x_{i+1 / 2}}\left\{\rho s_{1}\right\}\left(\rho, \rho u, \rho E, \rho s_{2}\right)\left(x, t^{n+1,=}\right) d x .
$$

This inequality is strict as soon as $\mathbf{v}_{i}^{n+1,=} \neq \mathbf{v}\left(x, t^{n+1,=}\right)$ for $x \in\left(x_{i-1 / 2}, x_{i+1 / 2}\right)$. Obviously, the (strict) inequality (3.7) met by $\left(\rho s_{1}\right)_{i}^{n+1,=}$ must be compared with the updating formula for $\left(\rho s_{2}\right)_{i}^{n+1,=}$ in (3.6):

$$
\left(\rho s_{2}\right)_{i}^{n+1,=}-\left(\rho s_{2}\right)_{i}^{n}+\frac{\Delta t}{\Delta x}\left\{\left\{\rho s_{2}\right\}\left(\omega\left(0^{+} ; \mathbf{v}_{i}^{n}, \mathbf{v}_{i+1}^{n}\right)-\left\{\rho s_{2}\right\}\left(\omega\left(0^{+} ; \mathbf{v}_{i-1}^{n}, \mathbf{v}_{i}^{n}\right)\right)\right\}=0,\right.
$$

where the equality just reflects the fact that $s_{2}$ is simply advected by the flow, contrary to $s_{1}$. This discrepancy in the rates of entropy dissipation, strictly negative versus zero, obviously results in a failure for satisfying the expected balance equation (2.12) at the discrete level. It is worth analysing further the reported discrepancy. To that end, let us recall the next estimate (see Coquel-LeFloch [5]):

$$
\begin{aligned}
& \frac{1}{\Delta x} \int_{x_{i-1 / 2}}^{x_{i+1 / 2}}\left(\left(\mathbf{v}\left(x, t^{n+1,=}\right)-\mathbf{v}_{i}^{n+1,=}\right),\left\{\overline{\nabla^{2} \rho s_{1}}\right\}_{i}^{n+1,=}\left(\mathbf{v}\left(x, t^{n+1,=}\right)-\mathbf{v}_{i}^{n+1,=}\right)\right) d x \\
& \quad=O(1)\left(\left\|\mathbf{v}_{i+1}^{n}-\mathbf{v}_{i}^{n}\right\|^{2}+\left\|\mathbf{v}_{i}^{n}-\mathbf{v}_{i-1}^{n}\right\|^{2}\right)
\end{aligned}
$$

where $O(1)$ is related to the convexity modulus of $\left\{\nabla^{2} \rho s_{1}\right\}(\mathbf{v})$. Therefore in the region of smoothness of the solution, the above term is small but achieves large negative values across shock discontinuities. Notice that this error term clearly increases with the strength of the shocks.

Of course, this negative result cannot be bypassed when using instead of the exact Godunov scheme a relevant entropy-satisfying approximate Riemann solver (see Hou-LeFloch 11 in the scalar case). Nevertheless, a discrete inequality for the Lax entropy $\left\{\rho s_{1}\right\}(\mathbf{v})$ such as (3.7) is actually required for the forthcoming nonlinear correction step to take place.

With this respect, we end this paragraph when proving the desirable stability estimates satisfied by (3.6) that will be preserved in the nonlinear projection procedure. A discrete entropy inequality for $\left\{\rho s_{1}\right\}_{i}^{n+1,=}$, distinct from (3.7), enters the following statement and will be motivated just hereafter:

Proposition 3.1. Under the CFL condition (3.5), the Lax entropy pair $\left(\rho s_{1}, \rho s_{1} u\right)$ obeys the following discrete entropy inequality:

$$
\left\{\rho s_{1}\right\}\left(\mathbf{v}_{i}^{n+1,=}\right)-\left(\rho s_{1}\right)_{i}^{n}+\frac{\Delta t}{\Delta x}\left\{\left\{g_{\rho s_{1} u}\right\}_{i+1 / 2}^{n}-\left\{g_{\rho s_{1} u}\right\}_{i-1 / 2}^{n}\right\}=\mathcal{E}_{i}^{n} \leq 0
$$


where the numerical entropy flux $\left\{g_{\rho s_{1} u}\right\}_{i+1 / 2}^{n}$ differs from $\left\{\rho s_{1} u\right\}\left(\omega\left(0^{+} ; \mathbf{v}_{i}^{n}, \mathbf{v}_{i+1}^{n}\right)\right)$ and is defined in full symmetry with the Larrouturou's flux function $\left\{g_{\rho s_{2} u}\right\}_{i+1 / 2}^{n}=$ $\left\{\rho s_{2} u\right\}\left(\omega\left(0^{+} ; \mathbf{v}_{i}^{n}, \mathbf{v}_{i+1}^{n}\right)\right.$ [14 in (3.9) by the following formula with $j=1,2$ :

$$
\begin{aligned}
\left\{g_{\rho s_{j} u}\right\}_{i+1 / 2}^{n}= & (\rho u)\left(w\left(0^{+} ; \mathbf{v}_{i}^{n}, \mathbf{v}_{i+1}^{n}\right)\right) \\
& \times \begin{cases}\left(s_{j}\right)_{i}^{n} & \text { if }(\rho u)\left(w\left(0^{+} ; \mathbf{v}_{i}^{n}, \mathbf{v}_{i+1}^{n}\right)\right)>0, \\
\left(s_{j}\right)_{i+1}^{n} & \text { otherwise. }\end{cases}
\end{aligned}
$$

Next, the following maximum principles on the specific entropies $s_{1}$ and $s_{2}$ are satisfied (see Tadmor [25]):

$$
\begin{aligned}
& \left\{s_{1}\right\}\left(\mathbf{v}_{i}^{n+1,=}\right) \leq \max \left(\left\{s_{1}\right\}\left(\mathbf{v}_{i-1}^{n}\right),\left\{s_{1}\right\}\left(\mathbf{v}_{i}^{n}\right),\left\{s_{1}\right\}\left(\mathbf{v}_{i+1}^{n}\right)\right), \\
& \left(s_{2}\right)_{i}^{n+1,=} \leq \max \left(\left(s_{2}\right)_{i-1}^{n},\left(s_{2}\right)_{i}^{n},\left(s_{2}\right)_{i+1}^{n}\right),
\end{aligned}
$$

away from a vacuum. Consequently, both the energies $\left(\epsilon_{1}\right)_{i}^{n+1,=}$ and $\left(\epsilon_{2}\right)_{i}^{n+1,=}$ stay positive.

The interest in considering a numerical entropy flux for $\rho s_{1}$ in full symmetry with the numerical flux entering the update for $\rho s_{2}$ stems from the need of preserving the exact symmetry in the role played by $\rho s_{1}$ and $\rho s_{2}$ in the forthcoming discrete version of the balance equation (2.29d). In addition, discrete analogues of the entropy-like inequalities (2.32) will easily follow from the choice (3.11). The proof detailed below asserts that the discrete entropy dissipation rate $\mathcal{E}_{i}^{n}$ in (3.10) is in general strictly negative.

Proof. Let us first establish the unusual discrete entropy inequality (3.10)-3.11) when closely investigating the property of $\left\{s_{1}\right\}\left(\omega\left(x / t ; \mathbf{v}_{i}^{n}, \mathbf{v}_{i+1}^{n}\right)\right)$ in each of the Riemann solutions. In view of the nonlinearity assumption (2.5) satisfied by the two pressure laws $p_{j}\left(\tau, s_{j}\right)$, the self-similar solution $\omega\left(x / t ; \mathbf{v}_{L}, \mathbf{v}_{R}\right)$ of the Cauchy problem (2.26) with initial data $\mathbf{v}_{0}(x)=\mathbf{v}_{L}, x<0, \mathbf{v}_{R}, x>0$ can be seen to be made of at most four constant states, denoted in the natural ordering by $\mathbf{v}_{L}, \mathbf{v}_{L}^{\star}, \mathbf{v}_{R}^{\star}, \mathbf{v}_{R}$, and separated by three simple waves. In the PDE model (2.26), the specific entropy $\left\{s_{1}\right\}(\mathbf{v})$ is easily seen to be a Riemann invariant for the two extreme genuinely nonlinear fields: this quantity therefore stays constant across the associated rarefaction waves. Considering a discontinuity propagating with speed $\sigma$ and separating the states $\mathbf{v}^{-}, \mathbf{v}^{+}$, the entropy inequality (3.3) can be re-expressed as follows:

$$
m\left\{s_{1}\left(\mathbf{v}^{+}\right)-s_{1}\left(\mathbf{v}^{-}\right)\right\} \leq 0, \quad m=\rho^{-}\left(u^{-}-\sigma\right)=\rho^{+}\left(u^{+}-\sigma\right),
$$

with strict inequality in the case of the two extreme genuinely nonlinear fields. Then, classical considerations in the usual gas dynamic setting (see [11]) directly extend to the present model (2.26) to prove that the relative mass flux $m$ in (3.14) respectively satisfies $m>0$ for a 1 -shock solution, $m=0$ across a contact discontinuity and $m<0$ concerning a 3 -shock. The above considerations for smooth and discontinuous elementary waves clearly yield

$$
s_{1}\left(\mathbf{v}_{L}\right) \geq s_{1}\left(\mathbf{v}_{L}^{\star}\right), \quad s_{1}\left(\mathbf{v}_{R}^{\star}\right) \leq s_{1}\left(\mathbf{v}_{R}\right)
$$

and thus suffice to prove that

$$
\left\{s_{1}\right\}\left(\omega\left(x / t ; \mathbf{v}_{L}, \mathbf{v}_{R}\right)\right) \leq A\left(x / t ; \mathbf{v}_{L}, \mathbf{v}_{R}\right) \equiv \begin{cases}s_{1}\left(\mathbf{v}_{L}\right) & \text { if } x / t<u^{\star} \\ s_{1}\left(\mathbf{v}_{R}\right) & \text { otherwise }\end{cases}
$$


where $u^{\star}=u_{L}^{\star}=u_{R}^{\star}$ denotes the speed of the intermediate contact discontinuity. Next, observe that the self-similar function $A\left(x / t ; \mathbf{v}_{L}, \mathbf{v}_{R}\right)$ entering the right-hand side of (3.15) is nothing but a solution of the following transport equation:

$$
\partial_{t} A+u\left(\omega\left(x / t ; \mathbf{v}_{L}, \mathbf{v}_{R}\right)\right) \partial_{x} A=0
$$

and hence, also solves

$$
\partial_{t} \rho A+\partial_{x} \rho A u=0 .
$$

Equipped with this result, we propose to supplement the system (3.2) with the decoupled PDE (3.16) when prescribing at time $t^{n}, A\left(x, t^{n}\right)=\left(s_{1}\right)_{i}^{n}$, with $x \in$ $\left(x_{i-1 / 2}, x_{i+1 / 2}\right)$, so that (3.15) clearly yields under the CFL condition (3.5):

$$
\begin{aligned}
\rho_{i}^{n+1,=} \times\left\{s_{1}\right\}(\mathbf{v})_{i}^{n+1,=} & \leq \frac{1}{\Delta x} \int_{x_{i-1 / 2}}^{x_{i+1 / 2}}\left\{\rho s_{1}\right\}\left(\rho, \rho u, \rho E, \rho s_{2}\right)\left(x, t^{n+1,=}\right) d x \\
& \leq \frac{1}{\Delta x} \int_{x_{i-1 / 2}}^{x_{i+1 / 2}}\{\rho A\}\left(x, t^{n+1,=}\right) d x,
\end{aligned}
$$

where the first line is just the Jensen inequality (3.8). But since $(\rho A)(x, t)$ solves (3.16), calculations due to Larrouturou [14] immediately give

$$
\begin{aligned}
& \frac{1}{\Delta x} \int_{x_{i-1 / 2}}^{x_{i+1 / 2}}\{\rho A\}\left(x, t^{n+1,=}\right) d x \equiv \rho_{i}^{n+1,=} \times A_{i}^{n+1,=} \\
& =(\rho A)_{i}^{n}-\frac{\Delta t}{\Delta x}\left\{\{\rho A\}\left(\omega\left(0^{+} ; \mathbf{v}_{i}^{n}, \mathbf{v}_{i+1}^{n}\right)\right)-\{\rho A\}\left(\omega\left(0^{+} ; \mathbf{v}_{i-1}^{n}, \mathbf{v}_{i}^{n}\right)\right)\right\},
\end{aligned}
$$

where by construction $(\rho A)_{i}^{n}=\left(\rho s_{1}\right)_{i}^{n}$ and $\{\rho A\}\left(\omega\left(0^{+} ; \mathbf{v}_{i}^{n}, \mathbf{v}_{i+1}^{n}\right)\right)=\left\{g_{\rho s_{1} u}\right\}_{i+1 / 2}^{n}$ as defined in (3.11). The required discrete entropy inequality (3.10) thus follows from (3.17).

Next, the maximum principle reported in (3.12) is recovered here as a by-product of (3.17)- (3.18) (see also 25] for a related approach) arguing that the advected quantity $A$ obeys the following under the CFL condition (3.5) and away from vacuum (see [14] for the details):

$$
A_{i}^{n+1,=} \leq \max \left(A_{i-1}^{n}, A_{i}^{n}, A_{i+1}^{n}\right), \quad A_{i}^{n}=\left(s_{1}\right)_{i}^{n}, \quad i \in \mathbb{Z} .
$$

The companion inequality (3.13) follows from the above inequality with $s_{2}$ in place of $A$ since $s_{2}$ is also purely advected by the flow. To conclude, notice that the monotonicity property $\partial_{\epsilon_{j}} s_{j}\left(\tau, \epsilon_{j}\right)=-T_{j}<0$ inferred from (2.3) ensures in view of the maximum principles (3.12) - (3.13) that the specific energies $\epsilon_{j}, j=1,2$ are kept positive.

3.1.2. Second step: The second-order operator $\left(t^{n+1,=} \rightarrow t^{n+1,-}\right)$. The partial update $\mathbf{v}_{h}\left(x, t^{n+1,=}\right)$ (see (3.6) $)$ is evolved to the next time level $t^{n+1,-}$ when solving for $t \in(0, \Delta t)$ the Cauchy problem:

$$
\left\{\begin{array}{l}
\partial_{t} \rho=0 \\
\partial_{t} \rho u=\partial_{x}\left(\left(\mu_{1}+\mu_{2}\right) \partial_{x} u\right), \\
\partial_{t} \rho E=\partial_{x}\left(\left(\mu_{1}+\mu_{2}\right) u \partial_{x} u\right), \\
\partial_{t} \rho s_{2}=-\frac{\mu_{2}}{T_{2}}\left(\partial_{x} u\right)^{2}
\end{array}\right.
$$


with $\mathbf{v}_{h}\left(x, t^{n+1,=}\right)$ as initial data. To that end, to keep the CFL restriction (3.5) unchanged, we consider a time implicit scheme defined for $i \in \mathbb{Z}$ by

$$
\begin{aligned}
\rho_{i}^{n+1,-} & =\rho_{i}^{n+1,=}, \\
(\rho u)_{i}^{n+1,-} & =(\rho u)_{i}^{n+1,=}+\Delta t{\overline{\partial_{x}\left(\left(\mu_{1}+\mu_{2}\right) \partial_{x} u\right)_{i}}}_{i+1,-}, \\
(\rho E)_{i}^{n+1,-} & =(\rho E)_{i}^{n+1,=}+\Delta t{\overline{\partial_{x}\left(\left(\mu_{1}+\mu_{2}\right) u \partial_{x} u\right)_{i}}}_{i+1,-}, \\
\left(\rho s_{2}\right)_{i}^{n+1,-} & =\left(\rho s_{2}\right)_{i}^{n+1,=}-\Delta t{\overline{\frac{\mu_{2}}{T_{2}}\left(\partial_{x} u\right)^{2}}}_{i},
\end{aligned}
$$

with the following consistent finite difference formulae built from the time averaging $M^{n} u_{i}=\left(u_{i}^{n+1,-}+u_{i}^{n+1,=}\right) / 2, i \in \mathbb{Z}$ :

$$
\begin{aligned}
& \overline{\partial x}_{x}\left(\left(\mu_{1}+\mu_{2}\right) \partial_{x} u\right)_{i}^{n+1,-}=\frac{\mu_{1}+\mu_{2}}{\Delta x^{2}}\left(M^{n} u_{i+1}-2 M^{n} u_{i}+M^{n} u_{i-1}\right) \\
& {\overline{\partial_{x}\left(\mu_{1}+\mu_{2}\right) u \partial_{x} u_{i}}}^{n+1,-}=\frac{\mu_{1}+\mu_{2}}{2 \Delta x^{2}}\left(\left(M^{n} u_{i+1}\right)^{2}-2\left(M^{n} u_{i}\right)^{2}+\left(M^{n} u_{i-1}\right)^{2}\right) \\
& \left(\overline{\frac{\mu_{2}}{T_{2}}\left(\partial_{x} u\right)^{2}}\right)_{i}^{n+1,-} \\
& =\frac{\mu_{2}}{2{\overline{T_{2}}}_{i}^{n+1,-} \Delta x^{2}}\left(\left(M^{n} u_{i+1}-M^{n} u_{i}\right)^{2}+\left(M^{n} u_{i}-M^{n} u_{i-1}\right)^{2}\right) .
\end{aligned}
$$

In (3.21c), the averaged temperature ${\overline{T_{2}}}_{i}^{n+1,-}$ receives the following definition:

$$
{\overline{T_{2}}}_{i}^{n+1,-}=\left\{\begin{array}{l}
T_{2}\left(\frac{1}{\rho_{i}^{n+1,-}},\left(s_{2}\right)_{i}^{n+1,-}\right), \text { if }\left(s_{2}\right)_{i}^{n+1,-}=\left(s_{2}\right)_{i}^{n+1,=}, \\
-\frac{\epsilon_{2}\left(\frac{1}{\rho_{i}^{n+1,-}},\left(s_{2}\right)_{i}^{n+1,-}\right)-\epsilon_{2}\left(\frac{1}{\rho_{i}^{n+1,-},},\left(s_{2}\right)_{i}^{n+1,=}\right)}{\left(s_{2}\right)_{i}^{n+1,-}-\left(s_{2}\right)_{i}^{n+1,=}}, \text { otherwise. }
\end{array}\right.
$$

Let us stress that the first two discrete operators 3.21a , 3.21b preserve by construction the conservation property to be met by the unknowns $\rho u$ and $\rho E$ while, as required, the last operator (3.21c) always achieves a positive sign. Besides, the benefit of these formulae is twofold as pointed out below.

On the one hand, since the density is kept unchanged in this second step, the implicit equations (3.20b for defining $\left\{u_{i}^{n+1-}\right\}_{i \in \mathbb{Z}}$ are actually completely decoupled from (3.20c) and (3.20d). Therefore solving (3.20) just amounts in practice to solving a linear problem in the unknown $M^{n} u$ with a positive definite symmetric matrix (away from a vacuum). Then, the total energy $(\rho E)_{i}^{n+1,-}$ can be readily point-wisely computed. Next considering the update of the specific entropy $s_{2}$, the definition of the averaged temperature ${\overline{T_{2}}}_{i}^{n+1,-}$ in (3.22) implies that $\left(s_{2}\right)_{i}^{n+1,-}, i$ being fixed, solves in each cell:

$$
\begin{aligned}
& \epsilon_{2}\left(\frac{1}{\rho_{i}^{n+1,-}},\left(s_{2}\right)_{i}^{n+1,-}\right)=\epsilon_{2}\left(\frac{1}{\rho_{i}^{n+1,-}},\left(s_{2}\right)_{i}^{n+1,=}\right) \\
& \quad+\frac{\mu_{2} \Delta t}{2 \rho_{i}^{n+1,=} \Delta x^{2}}\left(\left(M^{n} u_{i+1}-M^{n} u_{i}\right)^{2}+\left(M^{n} u_{i}-M^{n} u_{i-1}\right)^{2}\right)>0
\end{aligned}
$$

The strict monotonicity property $\partial_{s_{2}} \epsilon_{2}\left(\tau, s_{2}\right)<0$ inferred from (2.3) ensures the existence and uniqueness of the expected solution. 
On the other hand, direct calculations based on the point-wise update (3.20c) for $(\rho E)_{i}^{n+1,-}$, the representation formula (3.23) for $\left(\epsilon_{2}\right)_{i}^{n+1,-}$ and an exact estimate for the kinetic energy $\rho^{n+1,=}\left(u_{i}^{n+1,-}-u_{i}^{n+1,=}\right) \times\left(u_{i}^{n+1,-}+u_{i}^{n+1,=}\right) / 2$ easily deduced from (3.20b-3.21b), yield in view of the total energy definition (2.1) the following update for $\left(\epsilon_{1}\right)_{i}^{n+1-}$ :

$$
\begin{aligned}
& \left(\epsilon_{1}\right)_{i}^{n+1,-}=\left(\epsilon_{1}\right)_{i}^{n+1,=}+ \\
& \quad \frac{\mu_{1} \Delta t}{2 \rho_{i}^{n+1,=} \Delta x^{2}}\left(\left(M^{n} u_{i+1}-M^{n} u_{i}\right)^{2}+\left(M^{n} u_{i}-M^{n} u_{i-1}\right)^{2}\right) .
\end{aligned}
$$

The next identity can thus be derived in exact symmetry with (3.20d $-(3.21 \mathrm{c})$ :

$$
\begin{aligned}
& \left\{\rho s_{1}\right\}\left(\mathbf{v}_{i}^{n+1,-}\right)=\left\{\rho s_{1}\right\}\left(\mathbf{v}_{i}^{n+1,=}\right) \\
& \quad-\frac{\mu_{1} \Delta t}{2 \Delta x^{2}{\overline{T_{1}}}_{i}^{n+1,-}}\left(\left(M^{n} u_{i+1}-M^{n} u_{i}\right)^{2}+\left(M^{n} u_{i}-M^{n} u_{i-1}\right)^{2}\right),
\end{aligned}
$$

when defining the averaged temperature ${\overline{T_{1}}}_{i}^{n+1,-}$ in full symmetry with ${\overline{T_{2}}}_{i}^{n+1,-}$ in (3.22). As a direct consequence of the reported exact symmetry, we get

$$
\begin{aligned}
& \mu_{2}{\overline{T_{1}}}_{i}^{n+1,-}\left\{\left(\rho s_{1}\right)_{i}^{n+1,-}-\left(\rho s_{1}\right)_{i}^{n+1,=}\right\} \\
& -\mu_{1} \bar{T}_{2}^{n+1,-}\left\{\left(\rho s_{2}\right)_{i}^{n+1,-}-\left(\rho s_{2}\right)_{i}^{n+1,=}\right\}=0 .
\end{aligned}
$$

This identity just expresses that in the second step devoted to the diffusive operator, the two entropies $\rho s_{1}$ and $\rho s_{2}$ can be kept in balance according to (2.12). This was precisely the goal of the finite difference scheme we have just described. Let us underline that other finite difference formulae are indeed possible but to our knowledge, such formulae systematically produce (strictly) negative error terms in the discrete entropy balance equation (3.24) for $\rho s_{1}$ and thus ruin the exact balance in (3.25).

3.1.3. Stability estimates of the classical method with $L^{2}$ projection. This paragraph gathers the stability estimates achieved by the splitting method, which we have described for approximating the solutions of the equivalent system (2.26). Such estimates will allow for the nonlinear correction procedure to take place in the next section and, in turn, the desirable ones will be inherited. Using the notation introduced in the previous sections, the next result directly follows from Lemma 3.1 and the identities (3.20d $-(3.21 \mathrm{c})$, (3.24):

Lemma 3.2. Under the CFL condition (3.5), the unknown $\rho s_{2}$ updates according to:

$$
\begin{aligned}
(3.26)\left(\rho s_{2}\right)_{i}^{n+1,-}-\left(\rho s_{2}\right)_{i}^{n}+\frac{\Delta t}{\Delta x}\left\{\left\{g_{\rho s_{2} u}\right\}_{i+1 / 2}^{n}\right. & \left.-\left\{g_{\rho s_{2} u}\right\}_{i-1 / 2}^{n}\right\} \\
& +\Delta t\left(\overline{\frac{\mu_{2}}{T_{2}}\left(\partial_{x} u\right)^{2}}\right)_{i}^{n+1,-}=0
\end{aligned}
$$

while the Lax entropy pair $\left(\rho s_{1}, \rho s_{1} u\right)$ obeys the discrete entropy inequality:

$$
\begin{aligned}
\left\{\rho s_{1}\right\}\left(\mathbf{v}_{i}^{n+1,-}\right)-\left(\rho s_{1}\right)_{i}^{n}+\frac{\Delta t}{\Delta x}\left\{\left\{g_{\rho s_{1} u}\right\}_{i+1 / 2}^{n}\right. & \left.-\left\{g_{\rho s_{1} u}\right\}_{i-1 / 2}^{n}\right\} \\
& +\Delta t\left(\overline{\mu_{1}} \bar{T}_{1}\left(\partial_{x} u\right)^{2}\right)_{i}^{n+1,-}=\mathcal{E}_{i}^{n} \leq 0 .
\end{aligned}
$$


The relations (3.26) and (3.27) immediately yield the following discrete analogue of (2.12):

$$
\begin{array}{r}
\mu_{2}{\overline{T_{1}}}_{i}^{n+1,-}\left(\left\{\rho s_{1}\right\}\left(\mathbf{v}_{i}^{n+1,-}\right)-\left(\rho s_{1}\right)_{i}^{n}+\frac{\Delta t}{\Delta x}\left\{\left\{g_{\rho s_{1} u}\right\}_{i+1 / 2}^{n}-\left\{g_{\rho s_{1} u}\right\}_{i-1 / 2}^{n}\right\}\right) \\
-\mu_{1}{\overline{T_{2}}}_{i}^{n+1,-}\left(\left(\rho s_{2}\right)_{i}^{n+1,-}-\left(\rho s_{2}\right)_{i}^{n}+\frac{\Delta t}{\Delta x}\left\{\left\{g_{\rho s_{2} u}\right\}_{i+1 / 2}^{n}-\left\{g_{\rho s_{2} u}\right\}_{i-1 / 2}^{n}\right\}\right) \\
=\mu_{2} \bar{T}_{i}^{n+1,-} \times \mathcal{E}_{i}^{n} \neq 0,
\end{array}
$$

which means nothing but a violation of the generalized jump condition (2.22). The nonlinear projection procedure precisely intends to cure this failure.

Remark 3.3. The proposed procedure, based on the variable $\left(\rho, \rho u, \rho E, \rho s_{2}\right)$ does not allow us to keep in balance $\rho s_{2}$ and $\rho s_{1}(\mathbf{v})$ according to the jump relation (2.22). To enforce for validity this jump relation, one should be tempted to derive a related algorithm but based on the variable $\left(\rho, \rho u, \rho s_{1}, \rho s_{2}\right)$. With this choice, the total energy $\rho E$ must be understood as a function $\{\rho E\}(\mathbf{w})$ of $\mathbf{w}$. Lemma 2.8 ensures that this function is strictly convex in $\mathbf{w}$. As a consequence, one would get at the end of the first step devoted to the approximation of the hyperbolic problem:

$$
\begin{gathered}
\{\rho E\}\left(\mathbf{w}_{i}^{n+1,=}\right)-\{\rho E\}\left(\mathbf{w}_{i}^{n}\right)+\frac{\Delta t}{\Delta x}\left(\left\{\left(\rho E+p_{1}+p_{2}\right) u\right\}\left(\mathbf{w}\left(0^{+} ; \mathbf{w}_{i}^{n}, \mathbf{w}_{i+1}^{n}\right)\right)\right. \\
\left.\left.-\left\{\left(\rho E+p_{1}+p_{2}\right) u\right\}\left(\mathbf{w}\left(0^{+} ; \mathbf{w}_{i-1}^{n}, \mathbf{w}_{i}^{n}\right)\right)\right\}\right) \leq 0
\end{gathered}
$$

with strict inequality in general. In other words, the total energy is not conserved. A correction procedure to restore the conservation of $\rho E$ is actually possible (see 1]) and it is close to the technique we describe in the $\mathbf{v}$ variable.

To assess the feasibility and the relevance of the forthcoming nonlinear correction procedure, the following stability estimates in the classical splitting method with $L^{2}$ projection are in order:

Theorem 3.4. Under the CFL restriction (3.5), the following discrete entropy inequalities are satisfied:

$$
\begin{aligned}
& \left\{\rho \phi_{j}\left(s_{j}\right)\right\}\left(\mathbf{v}_{i}^{n+1,-}\right)-\left(\rho \phi_{j}\left(s_{j}\right)\right)_{i}^{n} \\
& \quad+\frac{\Delta t}{\Delta x}\left\{\left\{g_{\rho \phi_{j}\left(s_{j}\right) u}\right\}_{i+1 / 2}^{n}-\left\{g_{\rho \phi_{j}\left(s_{j}\right) u}\right\}_{i-1 / 2}^{n}\right\} \leq 0, \quad j=1,2
\end{aligned}
$$

for any given increasing and convex smooth function $\phi_{j}: \mathbb{R} \rightarrow \mathbb{R}$. The numerical entropy flux function entering (3.29) is given by the Larrouturou's formula (3.11). Next, the following maximum principles on the specific entropies $s_{1}$ and $s_{2}$ hold true away from a vacuum:

$$
\left(s_{j}\right)_{i}^{n+1,-} \leq \max \left(\left(s_{j}\right)_{i-1}^{n},\left(s_{j}\right)_{i}^{n},\left(s_{j}\right)_{i+1}^{n}\right), \quad j=1,2 .
$$

As a consequence, the internal energies $\left(\epsilon_{1}\right)_{i}^{n+1,-}$ and $\left(\epsilon_{2}\right)_{i}^{n+1,-}$ are kept positive.

The inequalities (3.29) are of course discrete versions of the entropy-like inequalities (2.32) encoding in particular the maximum principles stated in (2.34) and recovered in (3.30). 
Proof of Theorem 3.4. Let us first establish (3.29). To that purpose, let us write from the discrete entropy inequalities stated in Lemma 3.2 with $j \in\{1,2\}$ fixed:

$$
\begin{aligned}
\rho_{i}^{n+1,-} \times\left\{s_{j}\right\}\left(\mathbf{v}_{i}^{n+1,-}\right) & \leq \rho_{i}^{n} \times\left(s_{j}\right)_{i}^{n} \\
& -\frac{\Delta t}{\Delta x}\left\{\left\{g_{\rho s_{j} u}\right\}_{i+1 / 2}^{n}-\left\{g_{\rho s_{j} u}\right\}_{i-1 / 2}^{n}\right\} .
\end{aligned}
$$

Next, observe after Larrouturou [14] that the formula for $\left\{g_{\rho s_{j} u}\right\}_{i+1 / 2}^{n}$ reads equivalently:

$$
\left\{g_{\rho s_{j} u}\right\}_{i+1 / 2}^{n}=\rho_{i+1 / 2}^{n} \times\left\{\left(u_{i+1 / 2}^{n}\right)_{+}\left(s_{j}\right)_{i}+\left(u_{i+1 / 2}^{n}\right)_{-}\left(s_{j}\right)_{i+1}\right\},
$$

where to shorten the notation, we have set

$$
\rho_{i+1 / 2}^{n}=\rho\left(\omega\left(0^{+} ; \mathbf{v}_{i}^{n}, \mathbf{v}_{i+1}^{n}\right)\right) \text { and } u_{i+1 / 2}^{n}=u\left(\omega\left(0^{+} ; \mathbf{v}_{i}^{n}, \mathbf{v}_{i+1}^{n}\right)\right),
$$

so that (3.31) can be rewritten (see 14] for the details):

$$
\begin{aligned}
& \rho_{i}^{n+1,-} \times\left\{s_{j}\right\}\left(\mathbf{v}_{i}^{n+1,-}\right) \leq \rho_{i}^{n+1,-} \\
& 32) \times\left\{\left(s_{j}\right)_{i}^{n}-\frac{\Delta t}{\Delta x}\left(v_{i+1 / 2}^{n}\right)^{-}\left(\left(s_{j}\right)_{i+1}^{n}-\left(s_{j}\right)_{i}^{n}\right)-\frac{\Delta t}{\Delta x}\left(v_{i-1 / 2}^{n}\right)^{+}\left(\left(s_{j}\right)_{i}^{n}-\left(s_{j}\right)_{i-1}^{n}\right)\right\},
\end{aligned}
$$

where we have introduced

$$
\begin{aligned}
& \left(v_{i+1 / 2}^{n}\right)^{-}=\rho_{i+1 / 2}^{n} \times\left(u_{i+1 / 2}^{n}\right)_{-} / \rho_{i}^{n+1-} \leq 0, \\
& \left(v_{i-1 / 2}^{n}\right)^{+}=\rho_{i-1 / 2}^{n} \times\left(u_{i-1 / 2}^{n}\right)_{+} / \rho_{i}^{n+1-} \geq 0 .
\end{aligned}
$$

Hence, (3.32) yields away from a vacuum:

$$
\begin{aligned}
\left\{s_{j}\right\}\left(\mathbf{v}_{i}^{n+1,-}\right) & \leq\left(1-\frac{\Delta t}{\Delta x}\left(\left(v_{i-1 / 2}^{n}\right)^{+}-\left(v_{i+1 / 2}^{n}\right)^{-}\right)\right) \times\left(s_{j}\right)_{i}^{n} \\
& -\frac{\Delta t}{\Delta x}\left(v_{i+1 / 2}^{n}\right)^{-} \times\left(s_{j}\right)_{i+1}^{n}+\frac{\Delta t}{\Delta x}\left(v_{i-1 / 2}^{n}\right)^{+} \times\left(s_{j}\right)_{i-1}^{n},
\end{aligned}
$$

where after Larrouturou, the right-hand side is nothing but a convex decomposition of $\left(\left(s_{j}\right)_{i-1}^{n},\left(s_{j}\right)_{i}^{n},\left(s_{j}\right)_{i+1}^{n}\right)$ under the CFL restriction (3.5) (see again [14] for the details). We immediately infer from this convex decomposition the next inequality:

$$
\begin{aligned}
\phi_{j}\left(s_{j}\left(\mathbf{v}_{i}^{n+1,-}\right)\right) & \leq\left(1-\frac{\Delta t}{\Delta x}\left(\left(v_{i-1 / 2}^{n}\right)^{+}-\left(v_{i+1 / 2}^{n}\right)^{-}\right)\right) \times \phi_{j}\left(\left(s_{j}\right)\right)_{i}^{n} \\
& -\frac{\Delta t}{\Delta x}\left(v_{i+1 / 2}^{n}\right)^{-} \times \phi_{j}\left(\left(s_{j}\right)\right)_{i+1}^{n}+\frac{\Delta t}{\Delta x}\left(v_{i-1 / 2}^{n}\right)^{+} \times \phi_{j}\left(\left(s_{j}\right)\right)_{i-1}^{n},
\end{aligned}
$$

where we have successively used the increasing property and then the convexity property of $\phi_{j}$. It now suffices to multiply the inequality (3.34) by $\rho_{i}^{n+1,-}$ and to apply backward each of the above steps from (3.33) to (3.31) to get the expected inequality (3.29). The validity of the maximum principles stated in (3.30) simply follows from the convex decomposition put forward in (3.33). The required positivity of the specific energies is obtained using the same arguments as those developed in the proof of Proposition 3.1.

3.2. Nonlinear projection methods. According to the discrete balance equation (3.28), standard finite volume methods induce a too large numerical rate of entropy dissipation for $\rho s_{1}$ in comparison with the entropy dissipation rate of $\rho s_{2}$ that in turn precludes the satisfaction of (2.22). Here, we propose to add as an additional step to classical methods based on the $L^{2}$ projection, a nonlinear correction procedure, the so-called nonlinear projection step, the purpose of which is precisely to restore at the discrete level the correct balance between the two entropy-dissipation 
rates when enforcing (2.22) for validity. The overall method will be understood as a consistent method for approximating the solutions of the unusual equivalent formulation (2.29). The correction procedure is first grounded when proving existence and uniqueness of a solution in each of the local nonlinear problems to be solved (one per cell). Then several stability estimates are derived. The relevance of this fairly simple technique will be further assessed by the numerical experiments.

3.2.1. Third step: Nonlinear projection $\left(t^{n+1,-} \rightarrow t^{n+1}\right)$. In order to preserve the required conservation properties, let us define:

$$
\rho_{i}^{n+1}=\rho_{i}^{n+1,-}, \quad(\rho u)_{i}^{n+1}=(\rho u)_{i}^{n+1,-}, \quad(\rho E)_{i}^{n+1}=(\rho E)_{i}^{n+1,-} .
$$

Then to enforce for validity the generalized jump condition (2.21) at the discrete level, we propose to seek for $\left(\rho s_{2}\right)_{i}^{n+1}$ as a solution of

$$
\begin{aligned}
& \mu_{2}{\overline{T_{1}}}_{i}^{n+1,-}\left(\left\{\rho s_{1}\right\}\left(\mathbf{v}_{i}^{n+1}\right)-\left(\rho s_{1}\right)_{i}^{n}+\frac{\Delta t}{\Delta x}\left\{\left\{g_{\rho s_{1} u}\right\}_{i+1 / 2}^{n}-\left\{g_{\rho s_{1} u}\right\}_{i-1 / 2}^{n}\right\}\right) \\
& -\mu_{1}{\overline{T_{2}}}_{i}^{n+1,-}\left(\left(\rho s_{2}\right)_{i}^{n+1}-\left(\rho s_{2}\right)_{i}^{n}+\frac{\Delta t}{\Delta x}\left\{\left\{g_{\rho s_{2} u}\right\}_{i+1 / 2}^{n}-\left\{g_{\rho s_{2} u}\right\}_{i-1 / 2}^{n}\right\}\right)=0
\end{aligned}
$$

where in view of (3.35), one must read

$$
\left\{\rho s_{1}\right\}\left(\mathbf{v}_{i}^{n+1}\right)=\left\{\rho s_{1}\right\}\left(\rho_{i}^{n+1,-},(\rho u)_{i}^{n+1,-},(\rho E)_{i}^{n+1,-} ;\left(\rho s_{2}\right)_{i}^{n+1}\right),
$$

i.e. as a nonlinear function of solely $\left(\rho s_{2}\right)_{i}^{n+1}$. Of course, as soon as $\left(\rho s_{2}\right)_{i}^{n+1}$ does exist, then $\left(\rho s_{1}\right)_{i}^{n+1}$ updates according to (3.37).

The scalar nonlinear problem (3.36) is built in each cell from the numerical entropy fluxes and the averaged temperatures respectively involved in the first and second steps. The discrete equation (3.36) thus gives rise to a consistent discretisation of (2.29d), provided that it can be uniquely solved in $\left(\rho s_{2}\right)_{i}^{n+1}$. This is the matter of the following statement, the proof of which heavily relies on the property of the (approximate) Riemann solver in the first step being entropy dissipative with respect to the Lax entropy pair $\left(\rho s_{1}, \rho s_{1} u\right)$ :

Theorem 3.5. Under the thermodynamic assumptions (2.2)-(2.4) and the CFL condition (3.5), the nonlinear projection step (3.36) built from step 1 and step 2 is uniquely solvable in $\left(\rho s_{2}\right)_{i}^{n+1}$ for each $i \in \mathbb{Z}$. In addition, the following stability estimates are in order:

$$
\left\{\rho s_{1}\right\}\left(\mathbf{v}_{i}^{n+1}\right) \geq\left\{\rho s_{1}\right\}\left(\mathbf{v}_{i}^{n+1,-}\right) \quad \text { and } \quad\left(\rho s_{2}\right)_{i}^{n+1} \leq\left(\rho s_{2}\right)_{i}^{n+1,-} .
$$

The proof of this result is postponed to the end of the present section. Let us emphasize that Theorem [3.5 stays valid as soon as any given entropy-satisfying approximate Riemann solver is involved in the first step in place of the Godunov method.

Remark 3.6. The above existence result allows us to consider $\mathbf{w}_{i}^{n+1}=\left(\rho_{i}^{n+1}\right.$, $\left.(\rho u)_{i}^{n+1},(\rho E)_{i}^{n+1},\left(\rho s_{1}\right)_{i}^{n+1}\right)$ with $\left(\rho s_{1}\right)_{i}^{n+1}$ defined by (3.37), so that when understanding $\left\{\rho s_{2}\right\}$ as a function of $\mathbf{w},\left(\rho s_{1}\right)_{i}^{n+1}$ is seen to be the unique solution of 
the symmetric version of (3.36):

$$
\begin{gathered}
\mu_{2}{\overline{T_{1}}}_{i}^{n+1,-}\left(\left(\rho s_{1}\right)_{i}^{n+1}-\left(\rho s_{1}\right)_{i}^{n}+\frac{\Delta t}{\Delta x}\left\{\left\{\rho s_{1} u\right\}_{i+1 / 2}^{n}-\left\{\rho s_{1} u\right\}_{i-1 / 2}^{n}\right\}\right) \\
-\mu_{1}{\overline{T_{2}}}_{i}^{n+1,-}\left(\left\{\rho s_{2}\right\}\left(\mathbf{w}_{i}^{n+1}\right)-\left(\rho s_{2}\right)_{i}^{n}+\frac{\Delta t}{\Delta x}\left\{\left\{\rho s_{2} u\right\}_{i+1 / 2}^{n}-\left\{\rho s_{2} u\right\}_{i-1 / 2}^{n}\right\}\right)=0 .
\end{gathered}
$$

In other words, the two entropies $\left(\rho s_{1}\right)_{i}^{n+1}$ and $\left(\rho s_{2}\right)_{i}^{n+1}$ are kept in exact symmetry. Furthermore due to the use of the Larrouturou formula (3.11) for defining the numerical entropy fluxes, it can be seen that working out the splitting method when privileging the unknown $\mathbf{w}$ in place of $\mathbf{v}$ gives rise to exactly (3.39) and thus (3.36). In this sense, the nonlinear projection procedure we propose restores at the discrete level the equivalence in the different formulations.

The next statement asserts that the nonlinear projection procedure (3.36) preserves the desirable stability properties of the prediction step. The estimates (3.38), essentially inferred from the use of an entropy-satisfying approximate Riemann solver in the first step, will turn out to be responsible for this central issue.

Theorem 3.7. Under the CFL restriction (3.5), the following discrete entropy inequalities are satisfied:

$$
\begin{aligned}
\left\{\rho \phi\left(s_{j}\right)\right\}\left(\mathbf{v}_{i}^{n+1}\right) & -\left(\rho \phi\left(s_{j}\right)\right)_{i}^{n} \\
& +\frac{\Delta t}{\Delta x}\left\{\left\{g_{\rho \phi_{j}\left(s_{j}\right) u}\right\}_{i+1 / 2}^{n}-\left\{g_{\rho \phi_{j}\left(s_{j}\right) u}\right\}_{i-1 / 2}^{n}\right\} \leq 0
\end{aligned}
$$

for any given increasing and convex function $\phi_{j}: \mathbb{R} \rightarrow \mathbb{R}$. Next, the following maximum principles on the specific entropies $s_{1}$ and $s_{2}$ are met:

$$
\left\{\begin{array}{l}
\left(s_{1}\right)_{i}^{n+1} \leq \max \left(\left(s_{1}\right)_{i-1}^{n},\left(s_{1}\right)_{i}^{n},\left(s_{1}\right)_{i+1}^{n}\right) \\
\left(s_{2}\right)_{i}^{n+1} \leq \max \left(\left(s_{2}\right)_{i-1}^{n},\left(s_{2}\right)_{i}^{n},\left(s_{2}\right)_{i+1}^{n}\right)
\end{array}\right.
$$

Both of the specific internal energies $\left(\epsilon_{1}\right)_{i}^{n+1}$ and $\left(\epsilon_{2}\right)_{i}^{n+1}$ are thus kept positive.

We now turn to proving Theorem 3.5

Proof. To shorten the notation, let us introduce:

$$
\begin{aligned}
& \left(\rho s_{1}\right)_{i}^{n+1, *}=\left(\rho s_{1}\right)_{i}^{n}-\frac{\Delta t}{\Delta x}\left\{\left\{g_{\rho s_{1} u}\right\}_{i+1 / 2}^{n}-\left\{g_{\rho s_{1} u}\right\}_{i-1 / 2}^{n}\right\} \\
& C_{i}^{n+1,-}=\frac{\mu_{2}{\overline{T_{1}}}_{i}^{n+1,-}}{\mu_{1} \bar{T}_{i}^{n+1,-}}>0
\end{aligned}
$$

so that (3.36) recasts as follows:

$$
\left(\rho s_{2}\right)_{i}^{n+1}-\left(\rho s_{2}\right)_{i}^{n+1,=}=C_{i}^{n+1,-}\left(\left\{\rho s_{1}\right\}\left(\mathbf{v}_{i}^{n+1}\right)-\left(\rho s_{1}\right)_{i}^{n+1, *}\right) .
$$

It turns out to be convenient to solve (3.44) in the auxiliary unknown $\mathcal{M}_{i}^{n+1}$ defined by the linear change of variable $C_{i}^{n+1,-} \mathcal{M}_{i}^{n+1}=\left(\rho s_{2}\right)_{i}^{n+1}-\left(\rho s_{2}\right)_{i}^{n+1,=}$, namely, to look for $\mathcal{M}_{i}^{n+1}$ as a solution of

$$
\begin{aligned}
\mathcal{M}_{i}^{n+1}= & \left\{\rho s_{1}\right\}\left(\rho_{i}^{n+1-},(\rho u)_{i}^{n+1-},(\rho E)_{i}^{n+1-}, C_{i}^{n+1,-} \mathcal{M}_{i}^{n+1}+\left(\rho s_{2}\right)_{i}^{n+1,=}\right) \\
& -\left(\rho s_{1}\right)_{i}^{n+1, *} .
\end{aligned}
$$


Existence and uniqueness of such a solution now follows from a direct study of the next function:

$$
\begin{aligned}
\theta(m) & =\left\{\rho s_{1}\right\}\left(\rho_{i}^{n+1,-},(\rho u)_{i}^{n+1,-},(\rho E)_{i}^{n+1,-}, C_{i}^{n+1,-} m+\left(\rho s_{2}\right)_{i}^{n+1,=}\right) \\
& -\left(\rho s_{1}\right)_{i}^{n+1, *}-m .
\end{aligned}
$$

Notice that (3.46) reads equivalently with little abuse in the notation

$$
\theta(m)=\rho_{i}^{n+1,-} \times\left\{s_{1}\right\}\left(\tau_{i}^{n+1,-},\left\{\epsilon_{1}\right\}\left(\tau_{i}^{n+1,-}, m\right)\right)-\left(\rho s_{1}\right)_{i}^{n+1, *}-m,
$$

where

$$
\begin{aligned}
\left\{\epsilon_{1}\right\}\left(\tau^{n+1,-}, m\right) & =(E)_{i}^{n+1,-}-\frac{\left(u_{i}^{n+1,-}\right)^{2}}{2} \\
& -\epsilon_{2}\left(\tau_{i}^{n+1,-}, C_{i}^{n+1,-} \tau_{i}^{n+1,-} m+\left(s_{2}\right)_{i}^{n+1,=}\right) .
\end{aligned}
$$

First, note that in view of the asymptotic assumption (2.4),$s_{2}$ runs from $-\infty$ to $+\infty$ so that the $m$-variable in (3.46) naturally runs over $\mathbb{R}$. Then, again in view of (2.4), $\lim _{m \rightarrow-\infty} \epsilon_{2}=0+$ yields from (3.48):

$$
\lim _{m \rightarrow-\infty}\left\{\epsilon_{1}\right\}\left(\tau_{i}^{n+1,-}, m\right)=(E)_{i}^{n+1,-}-\frac{\left(u_{i}^{n+1,-}\right)^{2}}{2}>\left\{\epsilon_{1}\right\}\left(\tau_{i}^{n+1,-}, 0\right)>0
$$

since $\left\{\epsilon_{j}\right\}\left(\tau_{i}^{n+1,-}, 0\right)=\left(\epsilon_{j}\right)_{i}^{n+1,-}>0$ with $j=1,2$ as stated in Theorem 3.4. The above finite and positive limit in turn ensures from (3.47) that

$$
\lim _{m \rightarrow-\infty} \theta(m)=+\infty
$$

We now turn to proving that $\theta(0) \leq 0$. This will prove that there exists at least one solution $\mathcal{M}_{i}^{n+1} \leq 0$ of $\theta(m)=0$. Let us indeed compute

$$
\theta(0)=\left\{\rho s_{1}\right\}\left(\rho_{i}^{n+1,-},(\rho u)_{i}^{n+1,-},(\rho E)_{i}^{n+1,-},\left(\rho s_{2}\right)_{i}^{n+1,=}\right)-\left(\rho s_{1}\right)_{i}^{n+1, *},
$$

and assume at this stage that

$$
\begin{aligned}
& \left\{\rho s_{1}\right\}\left(\rho_{i}^{n+1,-},(\rho u)_{i}^{n+1,-},(\rho E)_{i}^{n+1,-},\left(\rho s_{2}\right)_{i}^{n+1,=}\right) \\
& \leq\left\{\rho s_{1}\right\}\left(\rho_{i}^{n+1,-},(\rho u)_{i}^{n+1,-},(\rho E)_{i}^{n+1,-},\left(\rho s_{2}\right)_{i}^{n+1,-}\right) .
\end{aligned}
$$

This inequality will be derived shortly but is immediately seen to imply from (3.49) that

$$
\begin{aligned}
\theta(0) & \leq\left\{\rho s_{1}\right\}\left(\rho_{i}^{n+1,-},(\rho u)_{i}^{n+1,-},(\rho E)_{i}^{n+1,-},\left(\rho s_{2}\right)_{i}^{n+1,-}\right) \\
& -\left(\rho s_{1}\right)_{i}^{n}+\frac{\Delta t}{\Delta x}\left\{\left\{g_{\rho s_{1} u}\right\}_{i+1 / 2}^{n}-\left\{g_{\rho s_{1} u}\right\}_{i-1 / 2}^{n}\right\},
\end{aligned}
$$

where $\left(\rho s_{1}\right)_{i}^{n+1, *}$ has been given its definition (3.42). The expected conclusion $\theta(0) \leq 0$ is then a direct consequence of the discrete entropy inequality (3.27) satisfied by the pair $\left(\rho s_{1}, \rho s_{1} u\right)$ under the CFL condition (3.5). At last, in view of $\partial_{s_{2}}\left\{s_{1}\right\}(\mathbf{v})=-T_{2} / T_{1}<0$, the smooth function $\theta(m)$ is actually strictly decreasing: $(3.52)$

$$
\begin{aligned}
& \frac{d \theta(m)}{d m}=-1 \\
& -C_{i}^{n+1,-}\left\{\frac{T_{2}}{T_{1}}\right\}\left(\rho_{i}^{n+1,-},(\rho u)_{i}^{n+1,-},(\rho E)_{i}^{n+1-}, C_{i}^{n+1,-} m+\left(\rho s_{2}\right)_{i}^{n+1,=}\right)<0,
\end{aligned}
$$

and this guarantees the uniqueness of a solution $\mathcal{M}_{i}^{n+1}$ of (3.45). Let us complete the proof of the existence and uniqueness of a solution of (3.45) with $\mathcal{M}_{i}^{n+1} \leq 0$ 
when proving the estimate (3.50). To that end, observe that $(\rho, \rho u, \rho E)$ being fixed, the function $\left(\rho s_{2}\right) \rightarrow\left\{\rho s_{1}\right\}\left(\rho, \rho u, \rho E, \rho s_{2}\right)$ is strictly decreasing in view of $\partial_{s_{2}}\left\{s_{1}\right\}(\mathbf{v})=-T_{2} / T_{1}<0$. Next, the discrete entropy inequality (3.26) ensures that $\left(\rho s_{2}\right)_{i}^{n+1,-} \leq\left(\rho s_{2}\right)_{i}^{n+1,=}$ and the required result directly follows from the above monotonicity property.

Let us now turn to deriving the second estimate entering (3.38). To that purpose, let us introduce

$$
m_{i}^{n+1,-}=-\frac{\mu_{1} \Delta t}{2 \Delta x^{2}{\overline{T_{1}}}_{i}^{n+1,-}}\left(\left(M^{n} u_{i+1}-M^{n} u_{i}\right)^{2}+\left(M^{n} u_{i}-M^{n} u_{i-1}\right)^{2}\right) \leq 0,
$$

in order to first rewrite from (3.20d):

$$
\left(\rho s_{2}\right)_{i}^{n+1,-}=\left(\rho s_{2}\right)_{i}^{n+1,=}+C_{i}^{n+1,-} m_{i}^{n+1,-} .
$$

and then to re-express the discrete entropy inequality (3.27) under the form:

$$
\begin{aligned}
& \left\{\rho s_{1}\right\}\left(\rho_{i}^{n+1,-},(\rho u)_{i}^{n+1,-},(\rho E)_{i}^{n+1,-},\left(\rho s_{2}\right)_{i}^{n+1,=}+C_{i}^{n+1,-} m_{i}^{n+1,-}\right) \\
& \quad-\left(\rho s_{1}\right)_{i}^{n+1, *}=\mathcal{E}_{i}^{n}+m_{i}^{n+1,-}
\end{aligned}
$$

where $\left(\rho s_{1}\right)_{i}^{n+1, *}$ is given in (3.42) and $\mathcal{E}_{i}^{n} \leq 0$ denotes the numerical entropy dissipation rate put forward in Lemma 3.1. By the definition (3.46) of the function $\theta(m)$, 3.54) just recasts as

$$
\theta\left(m_{i}^{n+1,-}\right)=\mathcal{E}_{i}^{n} \leq 0 .
$$

The function $m \rightarrow \theta(m)$ being strictly decreasing (see (3.52)) with the property that $\theta\left(\mathcal{M}_{i}^{n+1}\right)=0$, the above estimate ensures that

$$
m_{i}^{n+1,-} \geq \mathcal{M}_{i}^{n+1} .
$$

The second inequality in (3.38) thus follows, since we deduce the required estimate

$$
\left(\rho s_{2}\right)_{i}^{n+1}-\left(\rho s_{2}\right)_{i}^{n+1,-}=C_{i}^{n+1,-}\left(\mathcal{M}_{i}^{n+1}-m_{i}^{n+1,-}\right) \leq 0
$$

in view of the representation formulae (3.53) and

$$
\left(\rho s_{2}\right)_{i}^{n+1}-\left(\rho s_{2}\right)_{i}^{n+1,=}=C_{i}^{n+1,-} \mathcal{M}_{i}^{n+1} .
$$

But this estimate now allows us to deduce the first inequality in (3.38), namely,

$$
\begin{aligned}
\left\{\rho s_{1}\right\}\left(\rho_{i}^{n+1,-},\right. & \left.(\rho u)_{i}^{n+1,-},(\rho E)_{i}^{n+1,-},\left(\rho s_{2}\right)_{i}^{n+1,-}\right) \\
& \leq\left\{\rho s_{1}\right\}\left(\rho_{i}^{n+1,-},(\rho u)_{i}^{n+1,-},(\rho E)_{i}^{n+1,-},\left(\rho s_{2}\right)_{i}^{n+1}\right),
\end{aligned}
$$

since as already claimed, the function $\left(\rho s_{2}\right) \rightarrow\left\{\rho s_{1}\right\}\left(\rho, \rho u, \rho E, \rho s_{2}\right)$ is strictly decreasing. This completes the proof.

The next technical result allows us to prove the stability estimates stated in Theorem 3.7 when adopting exactly the same steps as those developed in the course of the proof of Theorem 3.4 ;

Lemma 3.8. Under the CFL restriction (3.5), the following inequalities are preserved by the nonlinear projection procedure:

$$
\begin{aligned}
\left\{\rho s_{j}\right\}\left(\mathbf{v}_{i}^{n+1}\right) & \leq\left\{\rho s_{j}\right\}\left(\mathbf{v}_{i}^{n}\right) \\
& -\frac{\Delta t}{\Delta x}\left\{\left\{g_{\rho s_{j} u}\right\}_{i+1 / 2}^{n}-\left\{g_{\rho s_{j} u}\right\}_{i-1 / 2}^{n}\right\}, \quad j=1,2,
\end{aligned}
$$

where the numerical entropy flux function is of Larrouturou's type (3.11). 
The proof heavily makes use of the second estimate in (3.38), Theorem 3.5

$$
\left(\rho s_{2}\right)_{i}^{n+1} \leq\left(\rho s_{2}\right)_{i}^{n+1,-} .
$$

Let us underscore that its validity essentially stems from the nonpositive entropy dissipation rate $\mathcal{E}_{i}^{n}$ entering the estimate (3.55). This observation again highlights the interest in involving a relevant entropy-satisfying Riemann solver in the first step of the method.

Proof of Lemma 3.8. The required inequality (3.56) with $j=2$ then immediately follows from the estimate (3.57) and (3.29) in Theorem 3.4. It in turn implies the companion inequality with $j=1$ just by the definition (3.36) of the nonlinear projection procedure.

3.2.2. Consistency with two asymptotic cases. This paragraph briefly highlights the consistency of the nonlinear projection method with two systems in full conservation form that are asymptotically recovered in the regime of a vanishing ratio of viscosities: namely either $\mu_{1} / \mu_{2}$ or $\mu_{2} / \mu_{1}$ goes to zero but with $\mu_{1}+\mu_{2} \geq \mu_{0}>0$. These two regimes are of practical importance in all the turbulence models (see [20] and the references therein). The equivalent system (2.29) indeed strongly suggests that in such a limit, the PDEs under consideration should meet a purely conservative formulation. The following statement gives support to this observation (see [3] for a proof):

Theorem 3.9. In the limit $\mu_{2}$ goes to zero, $\mu_{1}>0$ being fixed, travelling wave solutions of the system (2.6) converge uniformly over $\mathbb{R}$ to travelling wave solutions of the following system of conservation laws:

$$
\left\{\begin{array}{l}
\partial_{t} \rho+\partial_{x} \rho u=0, \quad x \in \mathbb{R}, t>0, \\
\partial_{t} \rho u+\partial_{x}\left(\rho u^{2}+p_{1}+p_{2}\right)=\partial_{x}\left(\mu_{1} \partial_{x} u\right), \\
\partial_{t} \rho E+\partial_{x}\left(\rho E+p_{1}+p_{2}\right) u=\partial_{x}\left(\mu_{1} u \partial_{x} u\right), \\
\partial_{t} \rho s_{2}+\partial_{x} \rho s_{2} u=0, \\
\quad \text { with } \partial_{t} \rho s_{1}(\mathbf{v})+\partial_{x} \rho s_{1}(\mathbf{v}) u \leq 0 .
\end{array}\right.
$$

Conversely, if $\mu_{1}$ goes to zero, $\mu_{2}>0$ being fixed, travelling wave solutions of the system (2.6) converge uniformly over $\mathbb{R}$ to travelling wave solutions of the next system of conservation laws:

$$
\left\{\begin{array}{l}
\partial_{t} \rho+\partial_{x} \rho u=0, \quad x \in \mathbb{R}, t>0, \\
\partial_{t} \rho u+\partial_{x}\left(\rho u^{2}+p_{1}+p_{2}\right)=\partial_{x}\left(\mu_{2} \partial_{x} u\right), \\
\partial_{t} \rho E+\partial_{x}\left(\rho E+p_{1}+p_{2}\right) u=\partial_{x}\left(\mu_{2} u \partial_{x} u\right), \\
\partial_{t} \rho s_{1}+\partial_{x} \rho s_{1} u=0, \\
\quad \text { with } \partial_{t} \rho s_{2}(\mathbf{w})+\partial_{x} \rho s_{2}(\mathbf{w}) u \leq 0 .
\end{array}\right.
$$

Let us observe that the nonlinear projection step (3.36) is by construction consistent with the above two asymptotic systems in full conservation form. Indeed, in the regime of a vanishing viscosity $\mu_{2}$, the algebraic relation (3.36) clearly gives the required asymptotic identities (see (3.58) ):

$$
\begin{aligned}
& \left(\rho s_{2}\right)_{i}^{n+1}=\left(\rho s_{2}\right)_{i}^{n}-\frac{\Delta t}{\Delta x}\left\{\left\{\rho s_{2} u\right\}_{i+1 / 2}^{n}-\left\{\rho s_{2} u\right\}_{i-1 / 2}^{n}\right\} \\
& \left(\rho \phi\left(s_{1}\right)\right)_{i}^{n+1}-\left(\rho \phi\left(s_{1}\right)\right)_{i}^{n}+\frac{\Delta t}{\Delta x}\left\{\left\{\rho \phi\left(s_{1}\right) u\right\}_{i+1 / 2}^{n}-\left\{\rho \phi\left(s_{1}\right) u\right\}_{i-1 / 2}^{n}\right\} \leq 0 .
\end{aligned}
$$


Conversely, the regime $\mu_{1} \rightarrow 0$ also gives the expected asymptotic behaviour in view of the symmetric form (3.39) of (3.36).

3.2.3. About variable viscosities. In practical applications, the viscosity laws are smooth functions of the state variable (see 20] for examples). All the above considerations are seen to extend to this framework when adopting in the second step of the method the following finite difference formulae, respectively, for

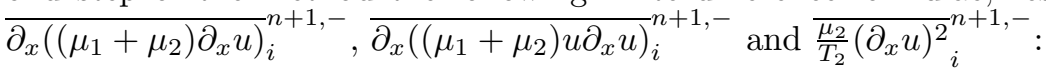

$$
\begin{aligned}
& \frac{1}{\Delta x^{2}}\left(\left(\mu_{T}\right)_{i+1 / 2}^{n}\left(M^{n} u_{i+1}-M^{n} u_{i}\right)-\left(\mu_{T}\right)_{i-1 / 2}^{n}\left(M^{n} u_{i}-M^{n} u_{i-1}\right)\right), \\
& \frac{1}{2 \Delta x^{2}}\left(\left(\mu_{T}\right)_{i+1 / 2}^{n}\left(\left(M^{n} u_{i+1}\right)^{2}-\left(M^{n} u_{i}\right)^{2}\right)-\left(\mu_{T}\right)_{i-1 / 2}^{n}\left(\left(M^{n} u_{i}\right)^{2}-\left(M^{n} u_{i-1}\right)^{2}\right)\right) \text {, } \\
& \frac{1}{2 \Delta x^{2}{\overline{T_{2}}}_{i}^{n+1,-}}\left(\left(\mu_{2}\right)_{i+1 / 2}^{n}\left(M^{n} u_{i+1}-M^{n} u_{i}\right)^{2}+\left(\mu_{2}\right)_{i-1 / 2}^{n}\left(M^{n} u_{i}-M^{n} u_{i-1}\right)^{2}\right) \text {, }
\end{aligned}
$$

with $\left(\mu_{T}\right)_{i+1 / 2}^{n}=\left(\mu_{1}\right)_{i+1 / 2}^{n}+\left(\mu_{2}\right)_{i+1 / 2}^{n}$ and ${\overline{T_{2}}}_{i}^{n+1,-}$ given by (3.22). The relevant extension of the nonlinear projection equation (3.36) then reads:

$$
\begin{aligned}
& {\overline{\mu_{2}}}_{i}^{n+1,-}{\overline{T_{1}}}_{i}^{n+1,-}\left(\left\{\rho s_{1}\right\}\left(\mathbf{v}_{i}^{n+1}\right)-\left(\rho s_{1}\right)_{i}^{n}+\frac{\Delta t}{\Delta x}\left\{\left\{\rho s_{1} u\right\}_{i+1 / 2}^{n}-\left\{\rho s_{1} u\right\}_{i-1 / 2}^{n}\right\}\right)- \\
& {\overline{\mu_{1}}}_{i}^{n+1,-} \bar{T}_{2}{ }_{i}^{n+1,-}\left(\left(\rho s_{2}\right)_{i}^{n+1}-\left(\rho s_{2}\right)_{i}^{n}+\frac{\Delta t}{\Delta x}\left\{\left\{\rho s_{2} u\right\}_{i+1 / 2}^{n}-\left\{\rho s_{2} u\right\}_{i-1 / 2}^{n}\right\}\right)=0,
\end{aligned}
$$

where for $j=1,2$ the averaged viscosities receive the following definitions:

$$
{\overline{\mu_{j}}}_{i}^{n+1,-}=\left\{\begin{array}{l}
\frac{\left(\mu_{j}\right)_{i+1 / 2}^{n}+\left(\mu_{j}\right)_{i-1 / 2}^{n},}{2} \quad \text { if } M^{n} u_{i+1}=M^{n} u_{i}=M^{n} u_{i-1}, \\
\frac{\left(\mu_{j}\right)_{i+1 / 2}^{n}\left(M^{n} u_{i+1}-M^{n} u_{i}\right)^{2}+\left(\mu_{j}\right)_{i-1 / 2}^{n}\left(M^{n} u_{i}-M^{n} u_{i-1}\right)^{2}}{\left(M^{n} u_{i+1}-M^{n} u_{i}\right)^{2}+\left(M^{n} u_{i}-M^{n} u_{i-1}\right)^{2}}, \text { otherwise. }
\end{array}\right.
$$

\section{THE METHOD FOR A GENERAL ENTROPY-SATISFYING NUMERICAL FLUX FUNCTION}

The numerical method is useful in that the nonlinear projection step may be used as a final corrector step to one's favourite underlying stable scheme and preserves its stability properties. We briefly summarize the proposed method for a general entropy-satisfying numerical flux function.

4.1. First step: Underlying first-order system $\left(t^{n} \rightarrow t^{n+1,=}\right)$. Let be given a consistent 2-points numerical flux function $\mathcal{F}: \Omega_{\mathbf{v}} \times \Omega_{\mathbf{v}} \rightarrow \mathbb{R}^{4}$ for the numerical approximation of the weak solutions of the hyperbolic problem (3.2) so as to define for all $i \in \mathbb{Z}$ :

$$
\begin{aligned}
& \rho_{i}^{n+1,=}=\rho_{i}^{n}-\frac{\Delta t}{\Delta x}\left(\mathcal{F}_{\rho}\left(\mathbf{v}_{i}^{n}, \mathbf{v}_{i+1}^{n}\right)-\mathcal{F}_{\rho}\left(\mathbf{v}_{i-1}^{n}, \mathbf{v}_{i}^{n}\right)\right), \\
& (\rho u)_{i}^{n+1,=}=(\rho u)_{i}^{n}-\frac{\Delta t}{\Delta x}\left(\mathcal{F}_{\rho u}\left(\mathbf{v}_{i}^{n}, \mathbf{v}_{i+1}^{n}\right)-\mathcal{F}_{\rho u}\left(\mathbf{v}_{i-1}^{n}, \mathbf{v}_{i}^{n}\right)\right), \\
& (\rho E)_{i}^{n+1,}=(\rho E)_{i}^{n}-\frac{\Delta t}{\Delta x}\left(\mathcal{F}_{\rho E}\left(\mathbf{v}_{i}^{n}, \mathbf{v}_{i+1}^{n}\right)-\mathcal{F}_{\rho E}\left(\mathbf{v}_{i-1}^{n}, \mathbf{v}_{i}^{n}\right)\right), \\
& \left(\rho s_{2}\right)_{i}^{n+1,=}=\left(\rho s_{2}\right)_{i}^{n}-\frac{\Delta t}{\Delta x}\left(\mathcal{F}_{\rho s_{2}}\left(\mathbf{v}_{i}^{n}, \mathbf{v}_{i+1}^{n}\right)-\mathcal{F}_{\rho s_{2}}\left(\mathbf{v}_{i-1}^{n}, \mathbf{v}_{i}^{n}\right)\right) .
\end{aligned}
$$


The finite volume method is assumed to be entropy-satisfying; namely, there exists a consistent 2-points numerical entropy flux function $\mathcal{F}_{\rho s_{1}}: \Omega_{\mathbf{v}} \times \Omega_{\mathbf{v}} \rightarrow \mathbb{R}$ such that, for all $i \in \mathbb{Z}$, the following inequality is valid under some CFL condition:

$$
\left\{\rho s_{1}\right\}\left(\mathbf{v}_{i}^{n+1,=}\right)-\left(\rho s_{1}\right)_{i}^{n}+\frac{\Delta t}{\Delta x}\left(\mathcal{F}_{\rho s_{1}}\left(\mathbf{v}_{i}^{n}, \mathbf{v}_{i+1}^{n}\right)-\mathcal{F}_{\rho s_{1}}\left(\mathbf{v}_{i-1}^{n}, \mathbf{v}_{i}^{n}\right)\right) \leq 0 .
$$

4.2. Second step: Second-order operator $\left(t^{n+1,=} \rightarrow t^{n+1,-}\right)$.

$$
\begin{aligned}
\rho_{i}^{n+1,-} & =\rho_{i}^{n+1,=}, \\
(\rho u)_{i}^{n+1,-} & =(\rho u)_{i}^{n+1,=}+\Delta t \overline{\partial_{x}\left(\left(\mu_{1}+\mu_{2}\right) \partial_{x} u\right)_{i}^{n+1,-}}, \\
(\rho E)_{i}^{n+1,-} & =(\rho E)_{i}^{n+1,=}+\Delta t \overline{\partial_{x}\left(\left(\mu_{1}+\mu_{2}\right) u \partial_{x} u\right)_{i}^{n+1,-},} \\
\left(\rho s_{2}\right)_{i}^{n+1,-} & =\left(\rho s_{2}\right)_{i}^{n+1,=}-\Delta t\left(\overline{\frac{\mu_{2}}{T_{2}}\left(\partial_{x} u\right)^{2}}\right)_{i}^{n+1,-},
\end{aligned}
$$

with the definitions introduced in (3.21) and (3.22).

The above two steps are referred to as an $L^{2}$-projection method.

4.3. Third step: Nonlinear projection $\left(t^{n+1,-} \rightarrow t^{n+1}\right)$. We keep unchanged

$$
\rho_{i}^{n+1}=\rho_{i}^{n+1,-}, \quad(\rho u)_{i}^{n+1}=(\rho u)_{i}^{n+1,-}, \quad(\rho E)_{i}^{n+1}=(\rho E)_{i}^{n+1,-},
$$

and we solve in $\left(\rho s_{2}\right)_{i}^{n+1}$ the following equation:

$$
\begin{aligned}
& \mu_{2}{\overline{T_{1}}}_{i}^{n+1,-}\left(\left\{\rho s_{1}\right\}\left(\mathbf{v}_{i}^{n+1}\right)-\left(\rho s_{1}\right)_{i}^{n}+\frac{\Delta t}{\Delta x}\left\{\left(\mathcal{F}_{\rho s_{1}}\right)_{i+1 / 2}^{n}-\left(\mathcal{F}_{\rho s_{1}}\right)_{i-1 / 2}^{n}\right\}\right) \\
& -\mu_{1}{\overline{T_{2}}}_{i}^{n+1,-}\left(\left(\rho s_{2}\right)_{i}^{n+1}-\left(\rho s_{2}\right)_{i}^{n}+\frac{\Delta t}{\Delta x}\left\{\left(\mathcal{F}_{\rho s_{2}}\right)_{i+1 / 2}^{n}-\left(\mathcal{F}_{\rho s_{2}}\right)_{i-1 / 2}^{n}\right\}\right)=0,
\end{aligned}
$$

where $\left(\mathcal{F}_{\rho s_{j}}\right)_{i+1 / 2}^{n}=\mathcal{F}_{\rho s_{j}}\left(\mathbf{v}_{i}^{n}, \mathbf{v}_{i+1}^{n}\right), j=1,2$.

This completes the description of the nonlinear projection method.

\section{NumERICAL EXPERIMENTS}

The ability of the discussed discrete methods, namely the usual splitting scheme with $L^{2}$ projection and the new nonlinear projection method, in the capture of stiff viscous shock layers for (2.6), is evaluated when testing their sensitivity in the prediction of the end states with respect to the mesh refinement. The pressure laws under consideration are those of two polytropic gases with distinct constant adiabatic exponent while the viscosities are taken to be positive constants. For the sake of comparison, the popular approach for approximating the solutions of the extended Navier-Stokes equations, briefly discussed below, is included in the benchmarks.

5.1. The popular approach for solving (2.6). Falling within the framework of splitting methods, the usual (if not systematic) approach for solving (2.6) consists in treating all the nonconservative products as being "source terms" so as to consider in a first step the following system in conservation form:

$$
\left\{\begin{array}{l}
\partial_{t} \rho+\partial_{x} \rho u=0, \quad x \in \mathbb{R}, t>0 \\
\partial_{t} \rho u+\partial_{x}\left(\rho u^{2}+p_{1}+p_{2}\right)=0 \\
\partial_{t} \rho E+\partial_{x}\left(\rho E+p_{1}+p_{2}\right) u=0 \\
\partial_{t} \rho \epsilon_{2}+\partial_{x} \rho \epsilon_{2} u=0
\end{array}\right.
$$


while in a next step, the "source term" $-p_{2} \partial_{x} u$ is to be given some ad hoc finite difference approximation. We refer the reader for instance to [15] and [20] for the details. Let us emphasizee the work by Forestier-Hérard-Louis 9] pointing out, on a theoretical ground, the violation of the positivity requirement for the internal energies. These authors have shown how to restore this positivity property when keeping the nonconservative product in (5.1) while defining it for shock solutions thanks to the Volpert product: $0.5\left(p_{2}^{-}+p_{2}^{+}\right) \times\left(u^{+}-u^{-}\right)$, i.e., regardless of the driving ratio of the viscosities.

5.2. Numerical results. The initial data of the Cauchy problems to be solved are made of two constant states, the discontinuity being located at $x=0$. In agreement with real life situations, the Reynolds number is set to $10^{6}$ in all of our experiments. The exact solutions of the associated Cauchy problem (2.6) therefore correspond to a stiff regularization of some Riemann solutions for the underlying hyperbolic system. The two extreme waves in our benchmarks will always correspond to compression waves. Thus, the exact solutions under consideration can be thought of as being made of two sharp travelling wave solutions separated by a contact wave. In the reference solution, displayed by a solid line hereafter, the end points of the two extreme waves are determined when integrating the ODE's system (2.15) governing the exact travelling wave solutions, for a prescribed state $\mathbf{v}$ in the past and a given velocity $\sigma$. To that end, we heavily use the property that the density $\rho(\xi)$ strictly increases with $\xi=x-\sigma t$ along the shock layer (see [1]). This allows us to re-express the ODE system (2.15) in the new space-like variable $\rho$ in a compact interval. We refer the reader to Berthon [1] for the technical details. Discrete solutions are systematically compared with this reference solution.

All the calculations described below have been performed according to the following strategy. An exact Roe-type linearization for system (3.2) yields an approximate Riemann solver to solve the first step (3.1.1) (see 6, 2] for the required formulae). Successive grid refinements, ranging from 100 to 2000 cells, are considered. The CFL number is fixed at the constant value 0.5.

Three test cases, labelled from A to C, are addressed. They are directly motivated by the three distinct regimes that underlie the fluid model under consideration and that are dictated by the amplitude of the viscosity ratio $\mu_{2} / \mu_{1}$. Namely, they involve a viscosity ratio which, when compared to unity, is successively small, close and large.

The associated initial data are defined in Table 1.

TABLE 1.

\begin{tabular}{|c|c|c|c|c|c|c|c|}
\hline Test & $\gamma_{1}$ & $\gamma_{2}$ & $\mu_{2} / \mu_{1}$ & $\rho$ & $u$ & $p_{1}$ & $p_{2}$ \\
\hline $\mathrm{A}$ & 1.4 & \multirow{2}{*}{1.6} & 0.01 & 1 & 1 & 1 & 0.6 \\
\cline { 5 - 8 } & & & & 1.05518 & -0.88895 & 0.15031 & 0.33869 \\
\hline $\mathrm{B}$ & 1.4 & \multirow{2}{*}{1.6} & 1 & 1 & 1 & 1 & 0.6 \\
\hline & & & & 1.92678 & -1.25451 & 2.74247 & 2.09561 \\
\hline $\mathrm{C}$ & 1.4 & \multirow{2}{*}{1.6} & \multirow{2}{*}{100} & 1 & 1 & 1 & 0.6 \\
\cline { 4 - 7 } & & & & 1.01595 & -0.93415 & 0.24142 & 0.15528 \\
\hline
\end{tabular}


Theorem 3.9 indicates that the solutions of the benchmarks $\mathrm{A}$ and $\mathrm{C}$ are asymptotically close to the solutions of systems of conservation laws. Namely, case A makes the entropy $\rho s_{2}$ to be merely advected by the flow while conversely, $\rho s_{1}$ inherits such a property in problem C. By contrast, the solution of problem B stays far away from these two asymptotic regimes. All the figures assess that the popular numerical strategy (5.1) grossly fails to properly restore the correct end states in all three regimes. Considering the $L^{2}$-projection method built from (2.29), the discrete solutions agree with the exact ones only in case A, as expected since here $\rho s_{2}$ is close to being a conservative variable. Such a property no longer holds for problems $\mathrm{B}$ and $\mathrm{C}$, and the discrete solutions exhibit large errors with the reference ones. Considering the nonlinear projection method, it produces approximate solutions in fairly good (if not excellent) agreement with the reference solutions while staying almost insensitive to the mesh refinement in the three investigated regimes.
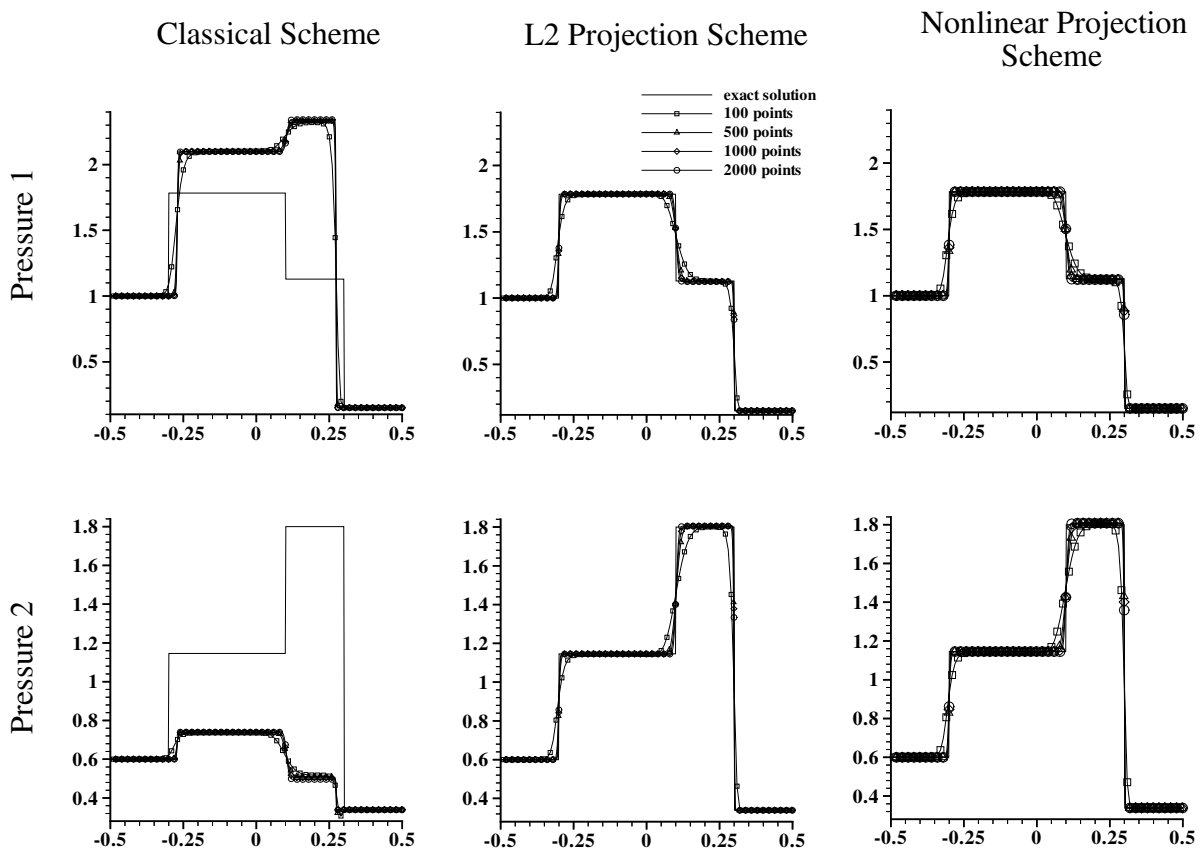

Figure 3. Problem A: $\mu_{2} / \mu_{1}<<1$ 


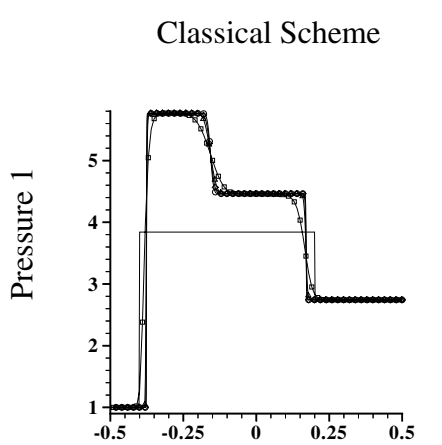

L2 Projection Scheme

Nonlinear Projection Scheme
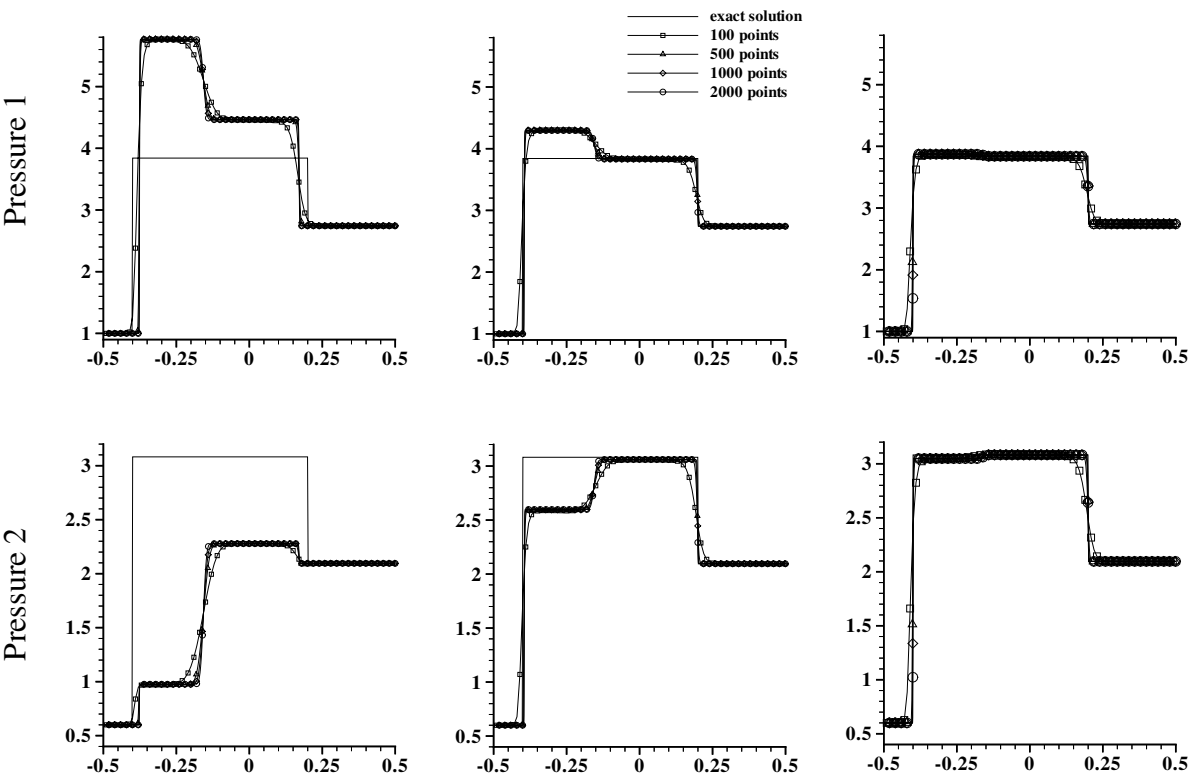

Figure 4. Problem B: $\mu_{2} / \mu_{1}=1$

Classical Scheme
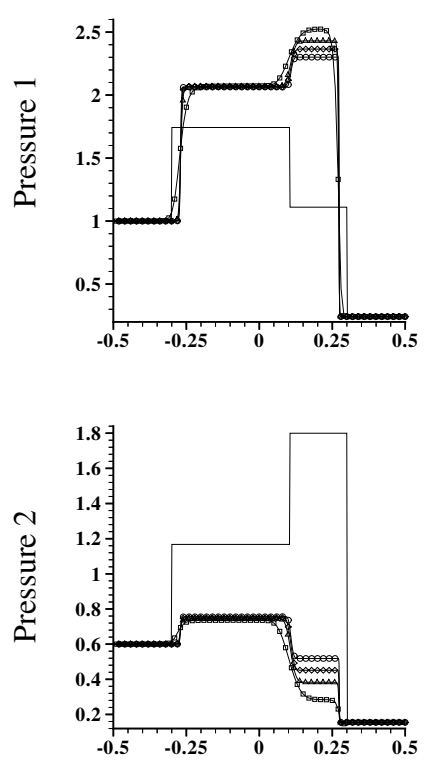

L2 Projection Scheme

Nonlinear Projection Scheme
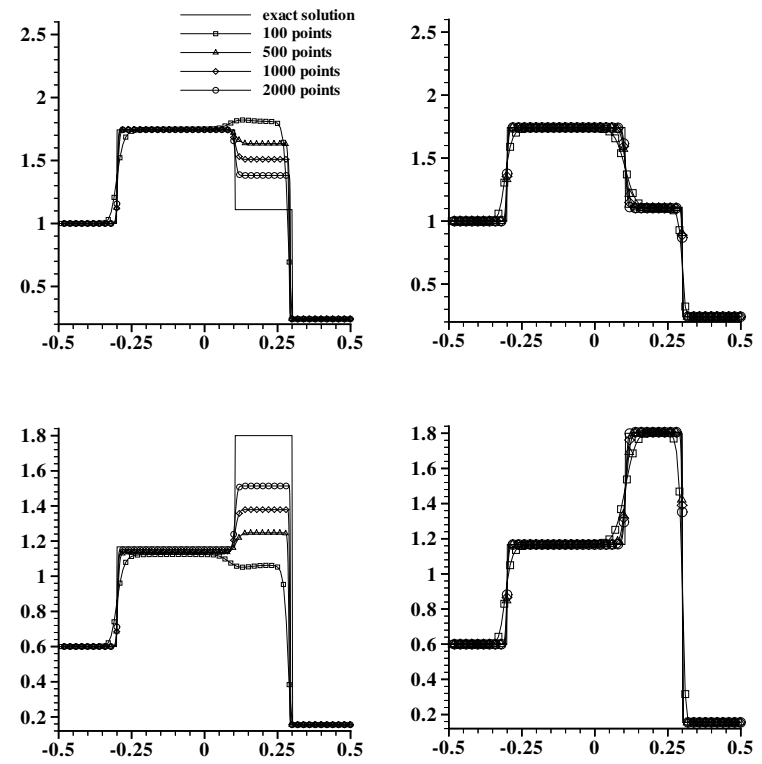

Figure 5. Problem C: $\mu_{2} / \mu_{1}>>1$ 


\section{REFERENCES}

[1] C. Berthon, Contributions to the numerical analysis of the compressible Navier-Stokes equations with two specific entropies. Applications to turbulent compressible flows. Ph.D. dissertation (in French) University Paris VI, 1999.

[2] C. Berthon And F. Coquel, Travelling wave solutions existence for multi-entropies NavierStokes equations, work in preparation, see also Proceedings of the 7th International Conference on Hyperbolic Problems, Zurich, 1998.

[3] C. Berthon and F. Coquel, About shock layers for compressible turbulent flow models, work in preparation.

[4] J.-F. Colombeau, A. Y. LeRoux, A. Noussaïr and B. Perrot, Microscopic profiles of shock waves and ambiguities in multiplications of distributions, SIAM J. of Numer. Anal., 26, No 4, 871-883 1989. MR1005514 (91c:35086)

[5] F. Coquel and P. LeFloch, Convergence of finite difference schemes for conservation laws in several space dimensions: the corrected antidiffusive flux approach, Math. Comp., 57, 169-210 1991. MR:1079010 (91m:65229)

[6] F. Coquel and C. Marmignon, A Roe-type linearization for the Euler equations for weakly ionized multi-component and multi-temperature gas, Proceedings of the AIAA $12^{\text {th }}$ CFD Conference, San Diego (USA) 1995.

[7] F. Coquel and B. Perthame, Relaxation of energy and approximate Riemann solvers for general pressure laws in fluid dynamics, SIAM J. of Numer. Anal., 35, No 6, 2223-2249 1998. MR1655844 (2000a:76129)

[8] G. Dal Maso, P. LeFloch and F. Murat, Definition and weak stability of nonconservative products, J. Math. Pures Appl., 74, 483-548 1995. MR 1365258 (97b:46052)

[9] A. Forestier, J.-M. Hérard and X. Louis, A Godunov-type solver to compute turbulent compressible flows, C.R. Acad. Sci. Paris, 324, Série I, No 8, pp. 919-926 1997. MR1450449 (98g:76048)

[10] D. GilbarG, The existence and limit behavior of the one-dimensional shock layer, Amer. J. Math., 73, 256-274 1951. MR0044315(13:401e)

[11] E. Godlewski and P.-A. Raviart, Hyperbolic systems of conservation laws, Applied Mathematical Sciences, Vol 118, Springer 1996. MR.1410987 (98d:65109)

[12] T. Y. Hou And P. G. LeFloch, Why nonconservative schemes converge to wrong solutions: error analysis, Math. of Comp., Vol 62, No 206, 497-530 1994. MR1201068 (94g:65093)

[13] S. Karni, Viscous shock profiles and primitive formulations, SIAM J. Numer. Anal., 29, No 6, 1592-1609 1992. MR:1191138 (93j:65163)

[14] B. Larrouturou, How to preserve the mass fractions positivity when computing compressible multi-component flows, J. Comput. Phys. 95, No 1, 59-84 1991. MR.1112315 (92k:76069)

[15] B. Larrouturou and C. Olivier, On the numerical approximation of the K-eps turbulence model for two dimensional compressible flows, INRIA report, No 15261991.

[16] P.D. Lax And B. Wendroff, Systems of conservation laws, Comm. Pure Appl. Math., Vol 13, 217-237 1960. MR0120774 (22:11523)

[17] P.G. LeFloch, Entropy weak solutions to nonlinear hyperbolic systems under nonconservative form. Comm. Part. Diff. Equa. 13, No 6, 669-727 (1988). MR0934378(89h:35194)

[18] F. R. Menter, Zonal two equation $(k-\omega)$ turbulence model for aerodynamic flows, 24th AIAA fluid dynamics conference, Orlando, (1993).

[19] F. R. Menter, Improved two equation $(k-\omega)$ turbulence model for aerodynamic flows, NASA Technical Report 103975, (1992).

[20] B. Mohammadi and O. Pironneau, Analysis of the k-Epsilon Turbulence Model, Research in Applied Mathematics, Masson, Paris, 1994. MR.1296252(95i:76048)

[21] P.-A. Raviart and L. Sainsaulieu, A nonconservative hyperbolic system modeling spray dynamics. Part 1. Solution of the Riemann problem, Math. Models Methods in Appl. Sci., 5, No 3, 297-333 1995. MR1330136 (96a:76096)

[22] P.L. Roe, Approximate Riemann solvers, parameter vectors and difference schemes, J. Comp. Phys., 43, 357-372 1981. MR0640362 (82k:65055)

[23] L. Sainsaulieu, Traveling waves solutions of convection-diffusion systems whose convection terms are weakly nonconservative, SIAM J. Appl. Math., 55, No 6, 1552-1576 1995. MR:1358789 (96m:65097) 
[24] B. R. Smith, A near wall model for the $(k-l)$ two equation turbulence model, 25th AIAA fluid dynamics conference, Colorado Springs, (1994).

[25] E. Tadmor, A minimum entropy principle in the gas dynamics equations, Appl. Numer. Math., No 2, 211-219 1986. MR0863987 (88a:76037)

[26] E. TADMOR, The numerical viscosity of entropy stable schemes for systems of conservation laws, I, Math. Comp., No 49, 91-103 1987. MR0890255 (88k:65087)

MAB, UMR 5466 CNRS, Université Bordeaux I, 351 cours de la libération, 33405 Talence Cedex, France

E-mail address: Christophe.Berthon@math.u-bordeaux1.fr

CNRS and Laboratoire Jacques-Louis Lions, UMR 7598, Tour 55-65, Université Pierre et Marie Curie, BC 187, 75252 Paris Cedex 05, France.

E-mail address: coquel@ann.jussieu.fr 